Canadian University Music Review

Revue de musique des universités canadiennes

\title{
Modèles linguistiques et analyse des structures musicales
}

\section{Jean-Jacques Nattiez}

Volume 23, numéro 1-2, 2003

URI : https://id.erudit.org/iderudit/1014517ar

DOI : https://doi.org/10.7202/1014517ar

Aller au sommaire du numéro

\section{Éditeur(s)}

Canadian University Music Society / Société de musique des universités canadiennes

\section{ISSN}

0710-0353 (imprimé)

2291-2436 (numérique)

Découvrir la revue

\section{Citer cet article}

Nattiez, J.-J. (2003). Modèles linguistiques et analyse des structures musicales. Canadian University Music Review / Revue de musique des universités

canadiennes, 23(1-2), 10-61. https://doi.org/10.7202/1014517ar

\section{Résumé de l'article}

$\mathrm{Au}$ cours de la seconde moitié $\mathrm{du} \mathrm{XX}^{\mathrm{e}}$ siècle, l'analyse musicale s'est tournée vers les modèles linguistiques d'inspiration structuraliste pour tenter de renouveler et de rendre plus explicites les méthodes traditionnelles. Dans cet article, qui est à la fois une bibliographie critique et un bilan épistémologique, Jean-Jacques Nattiez examine dans quel contexte musicologie et linguistique se sont rencontrées. Il présente tour à tour les applications du modèle phonologique, du modèle paradigmatique (avec une discussion des propositions et de la postérité de Ruwet) et les diverses grammaires musicales descriptives d'inspiration génératives qui ont été proposées. Il examine aussi bien les travaux qui portent sur la musique occidentale que sur les répertoires qu'étudient l'ethnomusicologie et les spécialistes de musique populaire.
All Rights Reserved (C Canadian University Music Society / Société de musique des universités canadiennes, 2004
Ce document est protégé par la loi sur le droit d'auteur. L'utilisation des services d'Érudit (y compris la reproduction) est assujettie à sa politique d'utilisation que vous pouvez consulter en ligne.

https://apropos.erudit.org/fr/usagers/politique-dutilisation/ 


\title{
MODÈLES LINGUISTIQUES ET ANALYSE DES STRUCTURES MUSICALES
}

\author{
Jean-Jacques Nattiez
}

\section{LA RENCONTRE DE LA MUSIQUE, DU STRUCTURALISME}

\section{ET DE LA LINGUISTIQUE}

On relie généralement à l'émergence de la sémiologie l'apparition de l'utilisation des modèles linguistiques dans l'analyse musicale. Cela est historiquement exact, à la condition de s'en tenir aux sphères culturelles françaises et italiennes, pour l'essentiel. Après la Deuxième Guerre mondiale, en effet, des chercheurs venus de différents horizons - les linguistes Roman Jakobson et Nicolas Ruwet, l'anthropologue Claude Lévi-Strauss, le critique littéraire Roland Barthes, le spécialiste du cinéma Christian Metz - assez proches les uns des autres par l'esprit mais témoignant de grandes différences entre eux quant à la méthodologie suivie et aux résultats empiriques obtenus, ont accrédité l'idée que la linguistique avait désormais le statut de « pilote des autres sciences sociales » (Ruwet 1963, 564).

Certes, il y avait dans la pensée européenne postérieure à la Seconde Guerre mondiale un intérêt général pour les réflexions concernant le langage. Citons l'ouvrage du philosophe Brice Parain, Recherches sur la nature et les fonctions du langage (1942) ou les écrits de l'esthéticien Mikel Dufrenne (1953, 1966, 1968), d'orientation phénoménologique, qui se demandait avec insistance si et comment l'art était langage. La question était présente également dans le domaine musical, comme l'a bien montré Enrico Fubini dans Musica e linguaggio nell'estetica contemporanea (1973). Un climat était donc favorable à la rencontre de la musique et de la linguistique. Mais celle-ci ne se serait sans doute pas produite, de manière non pas spéculative, mais empirique et concrète dans le champ de l'analyse musicale ${ }^{1}$, si on n'avait pas assisté à l'émergence du structuralisme, dans les domaines d'abord linguistiques, ensuite non linguistiques.

À la lumière des propositions théoriques du Cours de linguistique générale de Ferdinand de Saussure (1916), on constate le succès à la fois méthodologique et empirique de la phonologie ${ }^{2}$. On ne la confondra pas avec la phonétique.

1 Cette étude ne porte que sur l'analyse des structures musicales. C'est la raison pour laquelle, dans cet article, j'exclus l'examen des comparaisons de la musique et du langage. Même si cet exercice de sémiologie comparée est un préalable indispensable à l'application des modèles linguistiques à l'analyse d'un objet non linguistique comme la musique, cet axe d'investigation nous entraînerait très loin, et en deçà du XXe siècle. Citons cependant, à titre d'exemples très partiels : Austerlitz (1983), Bierwisch (1979), Harweg (1967), Nattiez (1975a, 2e partie), Pagnini (1974) et Springer (1956). Le problème de la sémantique, de la rhétorique et de la narrativité musicales en sont également exclus.

2D'un point de vue historique strict, la phonologie est née et s'est développée, à ses débuts, 
La phonétique se préoccupe de décrire physiquement et acoustiquement tous les aspects sonores du langage. La phonologie, elle, s'intéresse aux unités minimales propres à une langue : les phonèmes. D'une part, elle est capable de proposer des critères explicites et des règles menant à leur délimitation; en même temps, elle en propose un ordonnancement qualifié de structural, de sorte que la définition de ces unités, à l'inverse des descriptions infinies de la phonétique, peut être réduite à un faisceau de traits pertinents, souvent binaires selon l'école de Jakobson, ou en petit nombre (Troubetzkoy, Martinet). En voici un exemple simple : le tableau des consonnes du français dans lequel il suffit de deux traits pour caractériser chaque phonème (exemple 1).

\begin{tabular}{|c|c|c|c|c|c|c|c|c|c|}
\hline & $\begin{array}{l}\text { 丞 } \\
\text { 营 }\end{array}$ & 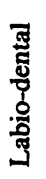 & 胥 & $\begin{array}{l}\text { 䓌 } \\
\text { 焉 }\end{array}$ & $\begin{array}{l}\text { 壳 } \\
\text { 胥 } \\
\text { 己 }\end{array}$ & 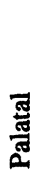 & 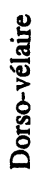 & 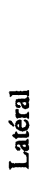 & 冤 \\
\hline Sourd & $p$ & $f$ & $t$ & s & š & & $\mathbf{k}$ & & \\
\hline Sonore & b & $\mathrm{v}$ & $d$ & $z$ & ž & & $g$ & & \\
\hline Nasal & $\mathbf{m}$ & & $\mathrm{n}$ & & & $\tilde{\mathbf{n}}$ & & & \\
\hline Non-nasal & & & & & & $i$ & & & \\
\hline Latéral & & & & & & & & 1 & \\
\hline Vibrant & & & & & & & & & $\mathrm{r}$ \\
\hline
\end{tabular}

Exemple 1. Tableau d'intégration phonologique des consonnes du français (Mounin 1968, 118)

Le succès empirique de la phonologie est aujourd'hui patent : Claude Hagège, pour caractériser la structure universelle des langues, a pu s'appuyer sur la description de 754 langues (Hagège 1982, 12). Elle dut d'emblée son succès épistémologique au fait qu'elle permettait de rompre avec l'historicisme et le comparatisme qui avait dominé les recherches linguistiques du XIXe siècle. Et le principe fondamental de l'explicitation des critères d'analyse, illustré par les règles pour la détermination des phonèmes dans les Principes de phonologie de N. S. Troubetzkoy (1939), allait passer dans les travaux du structuralisme linguistique américain qui ne se limitait pas à l'étude des phonèmes : l'école dite distributionnelle de Zellig Harris (1951) qui proposait des critères fondés sur l'environnement des unités constitutives du langage, qu'elles soient phonologiques, morphologiques ou syntaxiques. Découpage et définition d'unités, construction de règles d'analyse ou de règles descriptives vont être pour longtemps, et jusqu'à aujourd'hui, les deux pierres angulaires de l'analyse musicale d'inspiration linguistique.

Nul mieux que le philosophe Paul Ricœur n'a défini et résumé, même si c'était pour les critiquer du point de vue phénoménologique, les vertus du structuralisme.

indépendamment des idées de Saussure, mais le couple langue/parole sur lequel il insiste avec tant d'efficacité ne pouvait pas ne pas fournir une solide assise théorique à la différence entre la phonétique et la phonologie. 
Le type d'intelligibilité qui s'exprime dans le structuralisme triomphe dans tous les cas où l'on peut :

a) travailler sur un corpus déjà constitué, arrêté, clos et, en ce sens, mort;

b) établir des inventaires d'éléments et d'unités;

c) placer ces éléments ou unités dans des rapports d'opposition, de préférence d'opposition binaire; et

d) établir une algèbre ou une combinatoire de ces éléments et de ces couples d'opposition. (Ricœur 1969, 80)

Et l'on assista, sous la bannière conjuguée de la sémiologie et du structuralisme, à la fois au développement d'une philosophie structuraliste qui a pu s'étendre jusqu'à l'analyse de l'action sociale (Althusser) et de l'inconscient (Lacan), mais aussi à des tentatives d'importation des modèles linguistiques dans des domaines non linguistiques. Je n'aborderai pas ici les diverses formes de la pensée structuraliste dont François Dosse (1991-92) a pu écrire l'histoire avec bonheur. Je me contenterai de rappeler, avec Raymond Boudon, qu'il n'y a, d'un point de vue empirique, d'analyse structurale au sens rigoureux du terme, que si l'on se donne comme objectif de décrire par un ensemble de règles - ce sont elles qui sont constitutives de la structure - le fonctionnement immanent d'un objet considéré comme un système plus ou moins défini. En dernière instance, selon Boudon $(1968,103)$, et c'est un point de vue que je partage (Nattiez 2003, 57-59), le degré de précision et de validité d'une description structurale dépend de la combinaison de deux facteurs : la possibilité de vérifier la théorie de l'objet étudié, c'est-à-dire sa description structurale, et le caractère défini ou indéfini de l'objet-système. C'est dire qu'il y a des domaines, ou des parties de domaines, qui se prêtent mieux que d'autres à l'analyse structurale : en tant qu'objet-système, par exemple, une œuvre musicale n'a certainement pas le même statut que le contenu de l'inconscient...

Ce dernier point est important à considérer, car c'est lui qui explique que, du point de vue de la rigueur scientifique, certaines entreprises structuralistes aient mieux réussi que d'autres. Déjà au sein du langage, le succès de la phonologie n'allait pas nécessairement garantir celui de la sémantique structurale (Greimas), puisque l'intégration phonologique organisait une trentaine de phonèmes pour une langue donnée, alors que le lexique comporte un minimum de 3000 mots... Le problème allait rester de même nature lorsqu'il a été question de transposer les modèles (ou l'inspiration) de la linguistique à des domaines non linguistiques : l'analyse structurale des relations de parenté et des mythes (LéviStrauss), celle de la poésie (Jakobson, Ruwet) et du récit littéraire (Barthes, Brémond, Greimas). Saussure, dans le Cours de linguistique générale n'avait-il pas écrit : « La linguistique peut devenir le patron général de toute sémiologie, bien que la langue ne soit qu'un système particulier. " $(1916,101)^{3}$ ?

On comprend l'enthousiasme des débuts et le triomphalisme de certains y compris de l'auteur de ces lignes - au moment de l'émergence du structura-

3L'objectif de cet article est de parler de l'application au domaine musical des modèles linguistiques. C'est pourquoi je laisse de côté la justification de l'utilisation du mot « sémiologie » à propos de cette utilisation. Il sera seulement fait allusion ici et là à la théorie sémiologique tripartite (Molino, Nattiez). 
lisme et de la sémiologie, d'autant plus que leur apparition coïncidait avec la montée des aspirations révolutionnaires d'une nouvelle génération, et qui allaient culminer en 1968. Enfin on allait disposer d'une approche " structuralosémiologique » qui nous débarrasserait de l'historicisme régnant dans la vieille Université. Enfin on expliquerait les œuvres littéraires avec d'autres outils que la vieille érudition biographique. Enfin on ne se perdrait plus dans les méandres infinis des significations où se complaisaient les herméneutes. Fini l'impressionnisme du commentaire! En 1966, l'inventeur de la musique concrète, Pierre Schaeffer, poussait un cri d'alarme, bien révélateur de l'esprit du temps :

Dans son ensemble, l'abondante littérature consacrée aux sonates, quatuors et symphonies, sonne creux. Seule l'habitude peut nous masquer la pauvreté et le caractère disparate de ces analyses [...] Si toute explication se dérobe, qu'elle soit notionnelle, instrumentale ou esthétique, mieux vaudrait avouer, somme toute, que nous ne savons pas grand-chose de la musique. Et pis encore, que ce que nous en savons est de nature à nous égarer plutôt qu'à nous conduire. (Schaeffer 1966, 19)

Le terrain était mûr pour l'importation en musicologie de méthodes qui n'avaient pas honte de réclamer, dans la démarche, l'esprit de rigueur. Et de fait, c'est ce que la linguistique avait à offrir.

Ce n'est pas l'objet de cet article que d'évaluer les succès respectifs et relatifs du structuralisme dans les divers domaines auxquels il a été appliqué : entre l'analyse structurale d'un système phonologique par Martinet et celle d'une nouvelle de Balzac par Barthes, il y a des abîmes de différence, au plan de la méthodologie, des soubassements épistémologiques et des résultats. L'important, pour notre propos, c'est que ce bouillonnement tous azimuts, et bien souvent chaotique, ait introduit dans le secteur particulier de l'analyse musicale des approches fondées sur des prémisses nouvelles. C'est entre 1971 et 1976 que ce nouveau courant a d'abord retenu l'attention par une série intensive de publications : une série de numéros spéciaux de revue, notamment dans Musique en jeu, édités par l'auteur de ces lignes (Nattiez 1971, 1973a, 1973b, 1973c, 1975b, 1975c) relayés par un numéro de la Revue de musicologie (Lortat-Jacob 1975, Vaccaro 1975, C. Deliège 1975a), le recueil d'articles de Nicolas Ruwet, Langage, musique, poésie (1972), mes Fondements d'une sémiologie de la musique (Nattiez 1975a) et le livre de Gino Stefani Introduzione alla Semiotica della Musica (1976). Plusieurs articles viennent fêter les noces de la musicologie et de la linguistique (Ruwet 1967b, Nattiez 1972, 1973e). Même si ce courant ne retient plus autant l'attention aujourd'hui parce qu'il a perdu son caractère de nouveauté, il se prolonge et se poursuit, parfois brillamment. Comme l'écrivent Baroni, Dalmonte et Jacoboni dans un ouvrage capital avec lequel le $\mathrm{XX}^{\mathrm{e}}$ siècle s'est bien terminé, Le regole della musica : "Il est certain que, à l'époque post-structuraliste, les enthousiasmes injustifiés et les audaces théoriques insuffisamment motivées sont tombés; mais le thème n'a pas disparu de l'horizon musicologique » $(1999,43)^{4}$. En 1962, Nicolas Ruwet écrivait : « Le temps des

4 « Certo, in epoca post-strutturalistica sono caduti entusiasmi ingiustificati o azzardi teorici non 
déclarations de principe, des manifestes inauguraux, est terminé » $(1972,99)$. Quarante ans après, il est possible d'évaluer l'apport et la portée des résultats empiriques nombreux qui se sont accumulés.

S'il existe, dans le champ de l'analyse musicale, depuis la fin des années 1950 , un corpus copieux de recherches et d'études portant sur des œuvres et des styles spécifiques, c'est parce que la musique, comme objet, présentait des caractéristiques intrinsèques qui se prêtaient particulièrement bien aux exigences du structuralisme et des modèles linguistiques dans ce qu'ils avaient de plus rigoureux. Dans ce qui est peut-être le premier article qui témoigne de la rencontre entre le structuralisme et la musicologie, Célestin Deliège (1965) s'était efforcé de montrer tout ce qu'il y avait déjà de structuraliste dans des travaux de musicologie et d'analyse musicale antérieurs à l'irruption historique du structuralisme. Milan Kundera (1984) a même pu suggérer que, si le structuralisme avait trouvé naissance, durant l'entre-deux-guerres, en Tchécoslovaquie, c'était en raison de la place prépondérante que la musique occupait (et occupe toujours) dans la culture de son pays d'origine. Avant 1968, il est peu de compositeurs marquants qui n'auraient pas adhéré à la conception esthétique de la musique comme " forme en mouvement " (Hanslick 1854) ou à la conception sémiologique de la musique comme "système autotélique ", c'est-à-dire renvoyant à lui-même (Jakobson 1970). Stravinsky : « La musique est, par son essence, impuissante à exprimer quoi que ce soit [...]. L'expression n'a jamais été la propriété immanente de la musique » ([1935-36] 1971, 116). Varèse : « Ma musique ne peut exprimer autre chose qu'elle-même " ([1925] 1983, 41). Boulez : "La musique est un art non signifiant " ([1961] 1995, 492). Je ne rouvrirai pas ici la sempiternelle discussion sur le caractère formaliste ou expressif de la musique. Tous ceux qui m'ont lu attentivement savent la place importante que je réserve à la dimension sémantique de la musique (Nattiez 1975a, 129-93; 1987, chap. 5) et l'estime dans laquelle je tiens les recherches expérimentales d'un Michel Imberty en matière de sémantique musicale. Mais précisément, ce que la comparaison attentive du langage verbal et de la musique nous a appris, c'est que la signification en musique n'a pas le même statut que dans le langage. Lorsque j'écoute l'Appassionata, je n'entends pas Beethoven me dire : "Longtemps je me suis couché de bonne heure. " En termes plus abstraits : la musique est certes capable de véhiculer des significations affectives, émotives, imageantes, voire imitatives, mais ce n'est pas au niveau des unités porteuses de ces significations qu'elle est organisée syntaxiquement; c'est au niveau d'unités discrètes (discrétisées ou discrétisables), donc propres à un traitement structural rigoureux au sens de Boudon.

Or, ce principe reste valable même lorsque le recours aux modèles linguistiques chez les musicologues s'accomplit en dehors de la mouvance structuralosémiologique. Si j'ai insisté sur l'influence de la tentation linguistique dans les sciences humaines en la circonscrivant à la France et à l'Italie, c'est parce que, ailleurs, ou bien elle n'a pas eu, à cette époque, d'influence notable (je pense en

sufficientemente motivati, ma il tema non à affatto scomparso dall'orizzonte musicologico ». La traduction est de l'auteur du présent article. 
particulier aux pays de langue allemande, dominés par l'historicisme), ou parce que, notamment aux États-Unis, l'intérêt pour la linguistique est né d'une tradition épistémologique différente. On y était peu concerné, à cette époque, par les discussions subtiles de certains Européens sur la naissance et le développement de la sémiologie et de la sémio-linguistique. Ce qui fascinait, c'était l'efficacité concrète de certains modèles, notamment celui de la grammaire générative qui venait de naître à Boston avec Chomsky (1957) dont on attendait beaucoup du côté des applications pratiques, comme la traduction automatique. De plus, et il faudra un jour écrire en détail l'histoire de cette rencontre, le modèle de la grammaire générative présentait de fortes analogies avec celui de Schenker dont l'influence commençait à se faire sentir à peu près à la même date : la pensée schenkérienne pénètre véritablement aux États-Unis avec la parution, en 1952, de l'ouvrage de Felix Salzer, Structural Hearing : Tonal Coherence in Music.

Il est temps de quitter le terrain de la mise en situation historique et épistémologique de la rencontre entre musique et linguistique pour aborder chacun des trois grands modèles linguistiques qui ont inspiré les musicologues : le modèle phonologique, le modèle paradigmatique et le modèle génératif. Nous tenterons, tout au long de cet examen, d'évaluer ce que l'application de ces modèles aux œuvres et aux processus musicaux a pu apporter à l'analyse musicale ${ }^{5}$.

\section{LE MODÈLE PHONOLOGIQUE}

Même si, historiquement, c'est le succès de la phonologie qui a contribué à accréditer le rôle moteur que la linguistique pourrait jouer dans les sciences humaines, son importation dans l'analyse musicale n'a pas donné lieu à un grand nombre de travaux. Certes, c'est en faisant référence à elle que la sémiologie musicale s'est fait connaître en Europe d'un large public. Dans le $\mathrm{n}^{\circ} 5$ de Musique en jeu (1971), on trouvait la traduction d'articles plus anciens et quelque peu programmatiques de Jakobson (1932), Nettl (1958), Bright (1963) et un texte inédit du compositeur François-Bernard Mâche (1971), faisant appel àcertains éléments de la phonologie pour analyser une pièce de Varèse. Mais déjà à l'époque du Cercle linguistique de Prague, des chercheurs avaient songé à s'inspirer de la phonologie naissante pour approcher la musique d'un point de vue fonctionnel (Becking 1933; Sychra 1948).

En linguistique, le modèle phonologique a pour objectif de déterminer quels sons appartiennent en propre à une langue : le japonais ne distingue pas entre [1] et [r], le français distingue entre le [é] de " chantai » et le [è] de « chantais », l'allemand distingue entre le [ch] de "Kirche » et celui de "Kirsche ", l'anglais entre le [a] de " cat » et celui de " cut », etc. La procédure dite de commutation, c'est-à-dire le remplacement d'un son par un autre, permet de voir si les différences phonétiques sont pertinentes dans une langue particulière, c'est-àdire si elles permettent de distinguer des mots. Les règles de détermination des phonèmes, explicitant comment on met en œuvre la procédure de commutation,

5 Pour d'autres panoramas de l'utilisation des modèles linguistiques dans l'analyse, dans le contexte de la sémiologie musicale, on se reportera à Nattiez (1975a) et à Monelle (1992). 
pouvaient donc être adaptées à un problème classique de l'ethnomusicologie : quelles sont les hauteurs propres à un système musical? L'entreprise la plus aboutie est sans doute, à ce jour, celle de Vida Chenoweth présentée dans deux ouvrages : Melodic Perception and Analysis (1972) et The Usarufas and Their Music (1979). Elle y propose une méthodologie fondée sur cinq règles explicites (1972, 53-57; 1979, chap. 9), destinée à définir les unités scalaires d'un système étranger à notre langage tonal occidental et fondée sur la phonologie du linguiste américain Kenneth Pike (1954, 1967). La méthode est essentiellement distributionnelle et statistique. Par exemple : "Two similar etic units which are found in contrast in identical environments are two separate emic units 》 (Chenoweth 1972, 53). Ou encore, si un intervalle apparaît beaucoup moins utilisé qu'un autre qui en est proche acoustiquement, on peut vérifier systématiquement si cette rareté est due au contexte d'apparition de l'intervalle en question (par exemple, au début de la pièce, quand la voix n'est pas encore " chauffée " ou à la fin, quand elle est fatiguée) (exemple 2).

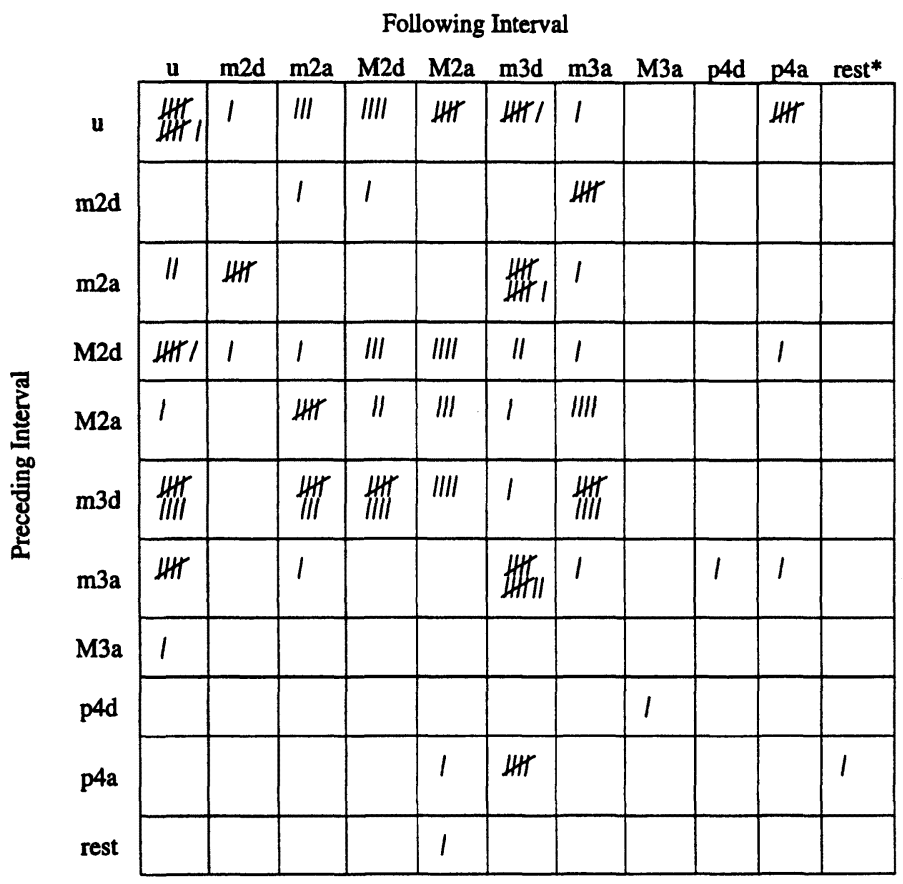

Exemple 2. Tableau des statistiques d'occurrences distributionnelles des intervalles, proposé pour un chant des Usarufas (Chenoweth 1972, 47)

Dans l'ouvrage de 1979, on trouve une riche collection de transcriptions " étiques » du corpus musical étudié, c'est-à-dire tel qu'il est entendu par une oreille occidentale, et de transcriptions " émiques ", c'est-à-dire reflétant le système musical propre aux autochtones. Dans un autre travail 
(1966), Chenoweth a pu démontrer que le système musical des Gadsups de Nouvelle-Guinée reposait sur des différences de quart de ton (signalées par un + , exemple 3).

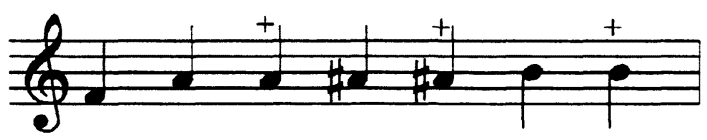

Exemple 3. Échelle musicale des Gadsups de NouvelleGuinée (Chenoweth 1966, 287)

Il serait injuste de prétendre que cette approche a connu une grande postérité, même si elle a pu inspirer une remarquable étude des intervalles dans un répertoire des Inuits du Caribou (Pelinski 1981a) et d'autres travaux ponctuels (Chandola 1970, 1977). Ruwet (1959) s'est inspiré de la distinction entre unités étiques et unités émiques pour dénoncer les " contradictions du langage sériel ». La phonologie a été utilisée aussi pour l'analyse des musiques électroacoustiques (Chiarucci 1973, Cogan 1984, Giomi et Ligabue 1998, Slawson $1985)^{6}$. Un chercheur brésilien s'est appuyé sur la phonologie de Jakobson pour analyser l'œuvre de Berio (Menezes 1993). La raison essentielle de la brièveté de cette bibliographie tient sans doute à la conjoncture scientifique. Alors que la question des échelles était à l'ordre du jour de l'ethnomusicologie depuis ses débuts jusqu'aux années 1960, les méthodes de Chenoweth émergent à l'intérieur de la décennie où l'ethnomusicologie se préoccupe de plus en plus d'expliquer la musique par son contexte culturel (Merriam 1964, Blacking 1973) et est moins soucieuse d'analyser des structures internes, de quelque nature que ce soit. La perspective ouverte par Chenoweth mérite d'être reprise, même si certains aspects de sa méthodologie devraient être révisés?

$\mathrm{Ce}$ qu'il faut sans doute retenir de la rencontre de la musicologie et de l'approche phonologique, c'est cette distinction d'ordre général et théorisée par Pike entre unités étiques et émiques. Ces néologismes sont dérivés des mots anglais désignant respectivement la phonétique et la phonologie : phonetics et phonemics. Ici encore, l'histoire de la transposition de ce couple conceptuel fertile de la linguistique à l'anthropologie, l'ethnomusicologie et l'analyse musicale mériterait d'être entreprise. Mais il y a dans cette distinction quelque chose de fondamental pour le musicologue. Alors que, au XIXe siècle, au moment des premiers balbutiements de l'ethnomusicologie, on n'hésite pas à transcrire les musiques de tradition orale en fonction du système tonal, voire à les harmoniser, le premier ethnomusicologue digne de ce nom, Alexan-

6 Pour un bilan critique, voir Roy 2003, 115-60.

7Elle prend comme unité de son investigation les intervalles; si elle s'appuyait sur les hauteurs, peut-être que les modalités d'application de certaines règles d'analyse seraient moins ambiguës. 
der Ellis (1885), invente le « cent », centième partie du demi-ton tempéré, pour analyser les échelles de la musique des Indes. Et c'est par crainte de tomber dans une forme sournoise d'ethnocentrisme musical que les chercheurs de l'école de Berlin (voir entre autres Hornbostel 1975) s'ingénient à noter la moindre différence entre les hauteurs enregistrées. L'idée que ces différences ne sont pas toutes pertinentes par rapport au système scalaire en jeu est une idée récente, mais décisive. Même si Simha Arom et ses collaborateurs (Collectif 1991), dans leurs recherches sur les échelles africaines, n'utilisent pas une méthode distributionnelle analogue à celle que propose Chenoweth, mais ont recours à des tests (consignés par ordinateur) auprès des informateurs sur le terrain concernant les techniques d'accordage, leur démarche repose fondamentalement sur la constatation que certaines différences de hauteurs sont pertinentes et que d'autres ne le sont pas et sur le fait qu'il est nécessaire de distinguer entre catégories étiques et catégories émiques.

Il convient de bien comprendre pourquoi le postulat structuraliste d'immanence fonctionne parfaitement pour découvrir les unités scalaires d'un système musical. Tout d'abord, parce que, tout comme les phonèmes d'une langue, elles sont en petit nombre : généralement inférieur à sept. Ensuite, parce que ces unités étant à la base du système, elles sont communes aux producteurs d'une pièce musicale et à ceux qui la perçoivent. Nous sommes en présence de ce que Molino appelle « le circuit court de la communication » (1989), par opposition au " circuit long » qui peut exister, selon d'autres paramètres ou d'autres niveaux de la production musicale, entre " producteurs 》 et " récepteurs ». Pour prendre un exemple extrême, entre la série des Structures pour deux pianos et ce qu'en perçoivent les auditeurs, ou plus exactement, ce qu'ils perçoivent de cette œuvre. La cassure entre le poïétique et l'esthésique, pour reprendre le jargon de la sémiologie musicale (Molino 1975, Nattiez 1975a, 1987) n'existant pas au niveau des unités scalaires, rien ne s'oppose à en fournir une description structurale immanente.

Dans ce qui est probablement, jusqu'à présent, la meilleure introduction à l'étude des musiques pop, Studying Popular Music, Richard Middleton examine tour à tour les trois grandes modèles linguistiques qui nous occupent ici. Commençant par la phonologie $(1990,176-83)$, il expose la différence entre catégories étiques et émiques, mais surtout, il indique que la procédure de commutation, signalée en commençant, a été systématiquement utilisée par Philip Tagg pour déterminer ce qu'il appelle les « musèmes ", c'est-à-dire les unités musicales minimales signifiantes. Middleton en fournit un exemple en analysant le début de la chanson des Beatles, «A Day in the Life » (180-82). Depuis, quinze ans de recherches auront permis à Tagg de proposer, dans un ouvrage majeur et dense (Tagg et Clarida 2003), une taxinomie générale des musèmes dont l'auteur montre plus d'une fois qu'elle peut être aussi utilsée pour l'analyse des musiques classiques « sérieuses". 


\section{LE MODÈLE PARADIGMATIQUE}

\section{Le modèle d'origine}

Dans son article historique de 1966, " Méthodes d'analyse en musicologie ", Ruwet écrivait :

Il m'a paru éclairant, dans l'étude des monodies, de reprendre un procédé que Claude Lévi-Strauss applique à l'analyse des mythes [...]. Les séquences équivalentes sont, autant que possible, écrites au-dessous les unes des autres, dans une même colonne, et le texte doit se lire, en faisant abstraction des blancs, de gauche à droite et de haut en bas. Ainsi, certains traits de structure sont immédiatement apparents, de même d'ailleurs que certaines ambiguîtés. $(1972,116-17)$

Et projetant « le principe d'équivalence de l'axe de sélection sur l'axe de la combinaison ", selon l'expression de Jakobson qui avait appliqué le même principe à l'analyse structurale de la poésie, Ruwet proposait de réécrire un Geisslerlied du XIVe siècle (exemple 4).

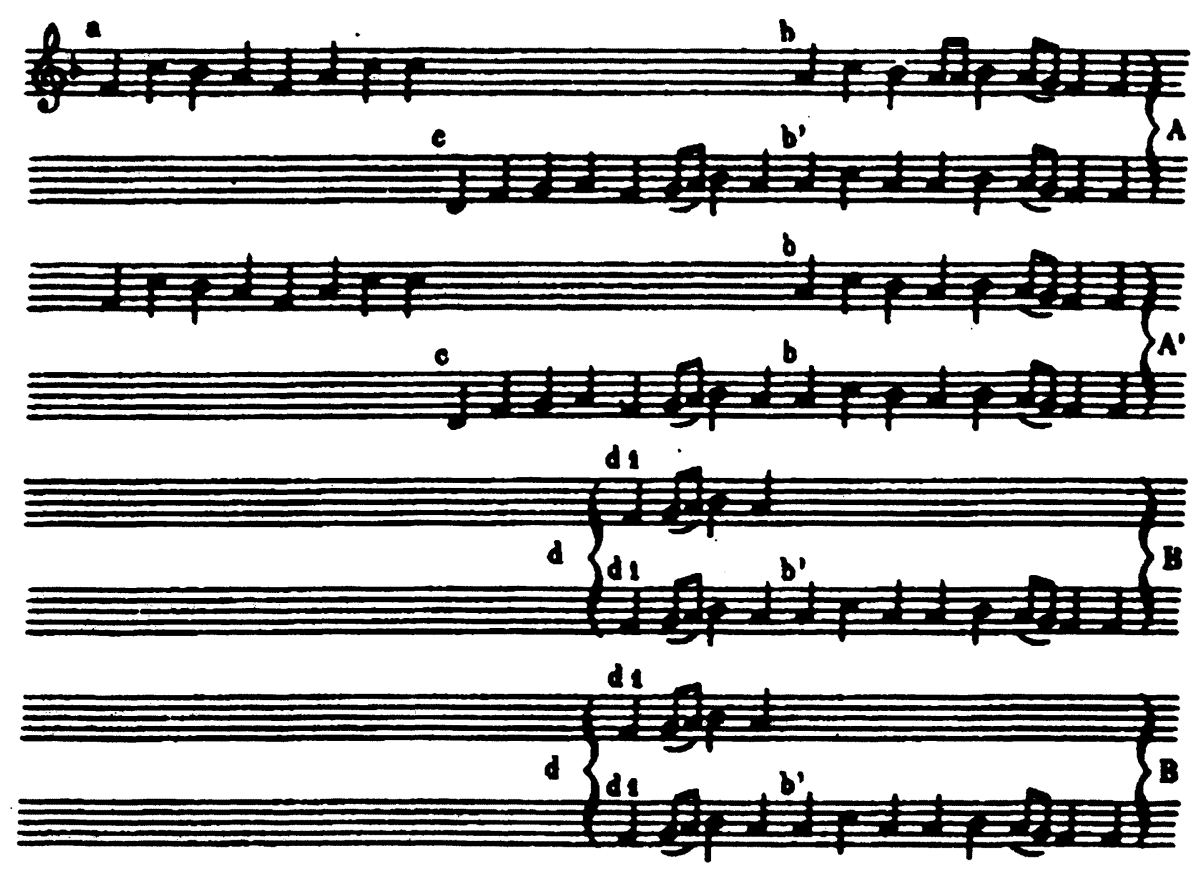

Exemple 4. Réécriture paradigmatique d'un Geisslerlied (Ruwet 1972, 116)

En opérant de cette manière, Ruwet s'inspirait d'une des grandes dichotomies fondatrices proposées par Saussure, celle qui distingue, dans un énoncé linguistique, entre les rapports syntagmatiques in praesentia et les rapports associatifs in absentia et qui allait conduire à la distinction entre syntagme et 
paradigme. En même temps, tout en se situant explicitement dans le cadre de la recherche sémiologique, il rencontrait les principes formalistes véhiculés par l'esthétique musicale du temps et que Jakobson nous semble avoir fort bien définis en ces termes:

Nicolas Ruwet [...] déclare que la syntaxe musicale est une syntaxe d'équivalences : les diverses unités sont dans des relations mutuelles d'équivalence multiforme. Cette affirmation suggère une réponse spontanée à la question complexe de la sémiosis musicale : plutôt que de viser quelque objet intrinsèque, la musique se présente comme un langage qui se signifie soi-même. Des parallélismes de structures construits et ordonnés différemment permettent à l'interprète de tout signans musical perçu immédiatement, de déduire et d'anticiper un nouveau constituant correspondant et l'ensemble cohérent formé par ces constituants. C'est précisément cette interconnexion des parties aussi bien que leur intégration dans un tout compositionnel qui fonctionne comme le signatum même de la musique. (Jakobson 1973, 99)

Si la musique était jeu de formes, il était possible de rendre compte, par des règles rigoureuses et selon des critères explicites, des relations entre des unités discrètes ou discrétisables.

Avec ses propositions méthologiques, Ruwet permettait en effet : 1) de situer syntagmatiquement chaque unité d'une monodie, analysée selon le critère de la dialectique entre répétition et transformation, par rapport à ses voisines; 2 ) de montrer leurs relations paradigmatiques avec des unités qui peuvent être placées beaucoup plus loin sur le syntagme; et 3 ) de montrer comment ces unités s'insèrent dans une organisation hiérarchisée. Mais si l'on trouve dans la littérature musicologique, avant Ruwet, des présentations analogues - chez Brailoiu et Rouget comme Ruwet l'a reconnu (1972, 104-5), chez Schoenberg (1943) comme me l'indiquait Célestin Deliège, ou chez plusieurs médiévistes comme l'a souligné Powers (1980) - la contribution essentielle de Ruwet, à une époque où on déplorait non sans raison le caractère impressionniste, voire par trop littéraire, de l'analyse musicale, a été d'appuyer la segmentation d'une pièce musicale sur une procédure d'analyse explicite que, pour l'analyse des monodies médiévales, Ruwet formulait, à l'exemple des meilleurs linguistes, en douze règles $(1972,112-15)$. En voici un exemple : " Notre "machine à repérer les identités élémentaires" parcourt la chaîne syntagmatique et repère les fragments identiques. On considère comme des unités du niveau I les séquences - les plus longues possibles - qui sont répétées intégralement, soit immédiatement après leur première émission, soit après l'intervention d'autres segments " (Ruwet 1972, 112). Cette notion d'explicitation, notre auteur ne la doit pas à Lévi-Strauss et Jakobson, mais à Harris qui, dans son important ouvrage de 1951, Structural Linguistics, un peu oublié aujourd'hui, mettait de l'avant la nécessité d'élaborer des " procédures de découverte » que le linguiste pourrait suivre, mais qui ont peut-être pour vertu essentielle, au-delà de leur aspect par trop mécaniste, d'offrir au chercheur des procédures de contrôle de sa démarche. En les introduisant 
enmusicologie, Ruwetsetrouvaitapporterunealternativeàl'impressionnisme généralisé du commentaire musical qui, à l'époque, en lassait plusieurs.

Le lien avec Harris nous conduit à une précision terminologique. On a parfois défini la méthode de Ruwet comme " distributionnelle » (Bent 1980, 377-78). Mais lorsqu'un chercheur propose un nouveau modèle d'analyse en s'inspirant de méthodes préexistantes, il ne retient pas nécessairement tout de chacune d'elle, et la technique distributionnelle proprement dite - celle qui consiste à définir une unité par son environnement syntagmatique - est absente de la méthodologie de Ruwet (alors qu'elle est au cœur de la démarche de Chenoweth) qui privilégie la dialectique de la répétition et des transformations entre unités d'un même paradigme. Du point de vue des influences, la paradigmatique de Ruwet est un hybride : à côté de la méthode paradigmatique proprement dite (adaptée de Lévi-Strauss et de Jakobson), de la notion de règle explicite inspirée de Harris, Ruwet emprunte aussi à l'analyse des mythes selon Lévi-Strauss, donc à l'anthropologie plutôt qu'à la linguistique, la manière de caractériser « structuralement " les unités musicales dégagées, comme on le voit dans son analyse du Prélude de Pelléas et Mélisande (Ruwet 1962). Lévi-Strauss ${ }^{8}$ y a même reconnu une anticipation de ses propres analyses de mythes dans Le cru et le cuit (1964).

On n'a pas suffisamment indiqué, à mon sens, que les règles explicites proposées par Ruwet reposaient sur ce que j'ai appelé ailleurs des principes transcendants (Nattiez 1987, 215), pas nécessairement énoncés par Ruwet en 1962 (mais on pourra les comparer aux éléments de " théorie sous-jacente " proposés dans Ruwet 1975, 26-27), et portant sur la nature de la musique : 1) Toute musique est constituée d'unités. Il est donc possible de les délimiter et de les définir, et de poser des règles pour les dégager. 2) Toute musique est hiérarchiquement organisée. Il est donc possible de répartir ces unités selon des niveaux distincts. 3) Toute musique révèle une dialectique de la répétition et de la variation. Il est donc possible, sur la base de la présentation paradigmatique, d'établir entre les unités des relations de transformation, et pour cela, il convient de dissocier les uns des autres les paramètres constitutifs de la substance musicale. Ruwet pratique ainsi le principe de l'autonomisation paramétrique sur lequel Meyer (1967, chap. 7; 1973), Molino (1975, 42-43) et Nattiez (1987, 180-82) ont insisté ultérieurement dans des contextes différents.

Comme on le voit, ces postulats ont un caractère universaliste, ce qui explique sans doute la postérité des propositions analytiques de Ruwet et l'étendue de leurs applications, parfaitement valables à la condition de respecter ce qu'on pourrait appeler les articulations naturelles des types de musiques étudiées. Mais en même temps que différents chercheurs appliquaient la méthode de Ruwet à de nouveaux objets, le modèle initial s'est modifié à la fois dans ses objectifs et dans son fonctionnement technique. 


\section{Applications des propositions analytiques de Ruwet et de la méthodologie paradigmatique}

Ruwet ne s'en est pas tenu lui-même à l'élaboration d'une méthode explicite d'analyse. Dans l'article de 1962 sur les duplications chez Debussy, il s'était proposé de raffiner une observation musicologique générale concernant la présence du procédé de duplication dans son œuvre, et il interprétait le jeu de structures dégagées dans le Prélude de Pelléas en termes fonctionnels, dérivés de l'analyse structurale des mythes comme on vient de l'indiquer. Et dans son analyse des monodies médiévales (1972, chap. 4), il a tenté de montrer comment induire des analyses structurales le mode utilisé.

Historiquement, à notre connaissance, c'est l'ethnomusicologue Simha Arom qui fut le premier à s'emparer du modèle de Ruwet. Son objectif était, sur la base de la paradigmatique, de proposer un nouveau système de " notation des monodies à des fins d'analyse " (1969), dans lequel il a démultiplié les points d'attaque de l'œuvre, donc les critères d'analyse paradigmatique. Il a également appliqué ces principes à un corpus Ngbaka-Ma'bo (Arom 1970). Un corpus de musique finlandaise a également fait l'objet d'une application stricte du modèle de Ruwet (Pekkilä 1987). Jusqu'ici, la démarche reste parfaitement immanente.

En 1976, Monique Brandily consacrait une étude paradigmatique fouillée à un seul chant du Tibesti (Tchad). Ici, la description n'est plus " purement » structurale : sans qu'elle utilise le terme, Brandily induit le modèle poiétique de la pièce en superposant chacune de ses phrases. Cette paradigmatique note à note (et non fondée sur des unités plus vastes comme chez Ruwet) permet de mettre en évidence un modèle, une structure mélodique de base qui n'est jamais actualisée comme telle, mais à partir de laquelle les différentes phrases du chant sont improvisées. La description immanente conduit donc à pénétrer le processus créateur. Entre 1974 et 1980, le Groupe de recherches en sémiologie musicale (G.R.S.M.) de l'Université de Montréal appliquait systématiquement la technique d'analyse à un vaste corpus de jeux de gorge inuits, et dans la première synthèse publiée à ce sujet (Nattiez 1983), l'analyse paradigmatique débordait aussi sur le poïétique. Il en va de même dans les analyses du volume consacré à l'Improvisation dans les musiques de tradition orale (Lortat-Jacob 1987) qui s'appuient explicitement sur le modèle de Ruwet.

C'est à Arom, encore une fois, qu'il revenait de proposer la démonstration la plus grandiose de l'efficacité de la méthode paradigmatique en l'appliquant systématiquement aux Polyphonies et polyrythmies instrumentales d'Afrique Centrale (1985) : avec ce vaste ouvrage, Arom offre la description, à mon avis la plus poussée, qu'on n'ait jamais donnée d'un système musical. Et même si l'auteur, dans son livre IV, croit que la démarche est restée immanente, il n'est que trop évident qu'elle lui a permis de déborder le seul niveau des structures pour déboucher sur des descriptions pertinentes du point de vue des processus de création (poïétique) et de perception (esthésique) (Nattiez 1990, 80-81). Donnons-en un exemple à propos du poïétique. L'exemple 5 offre les premiers paradigmes des variations effectuées par les cinq premières trompes d'un ensemble de 18 trompes Banda-Linda. 
Trompe $1:$ tété

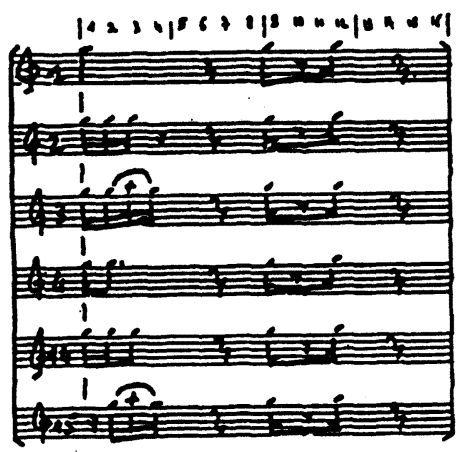

Trompe 3 : hā

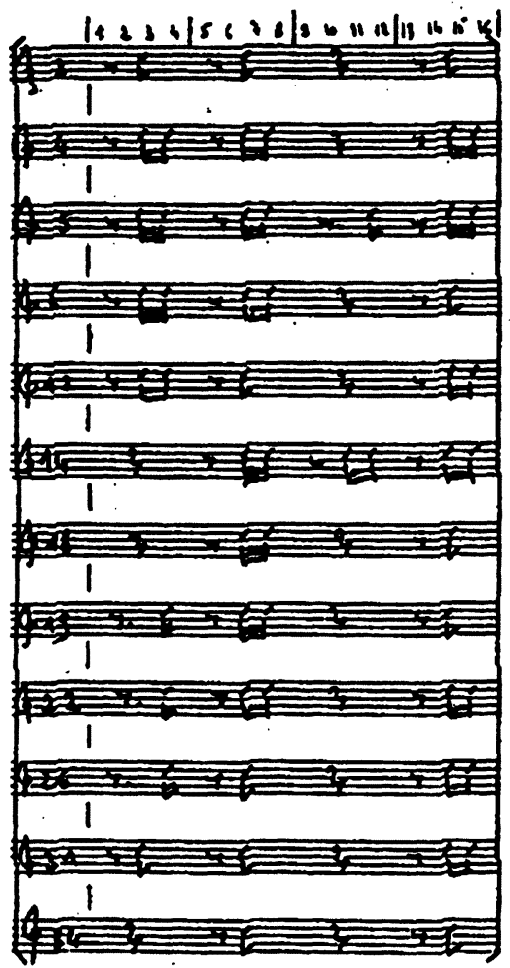

Trompe $2:$ tā

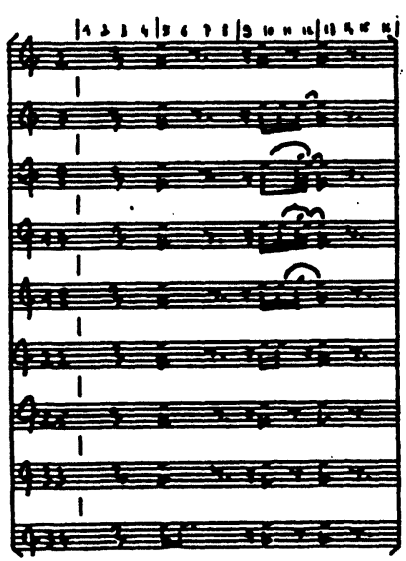

Trompe 4 : tútûlé

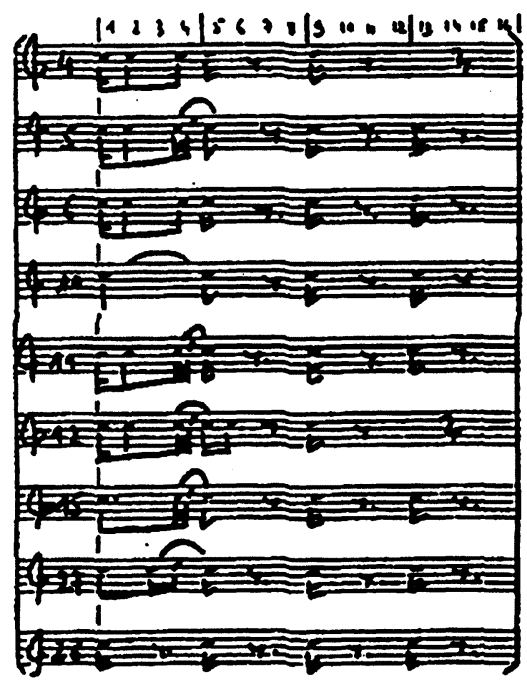

Exemple 5. Inventaire paradigmatique des unités exécutées par les cinq trompes BandaLinda dans la pièce ndàràjé baléndoro (Arom 1985, 544-45) 
Exemple 5-suite

Trompe 5 : bòngó

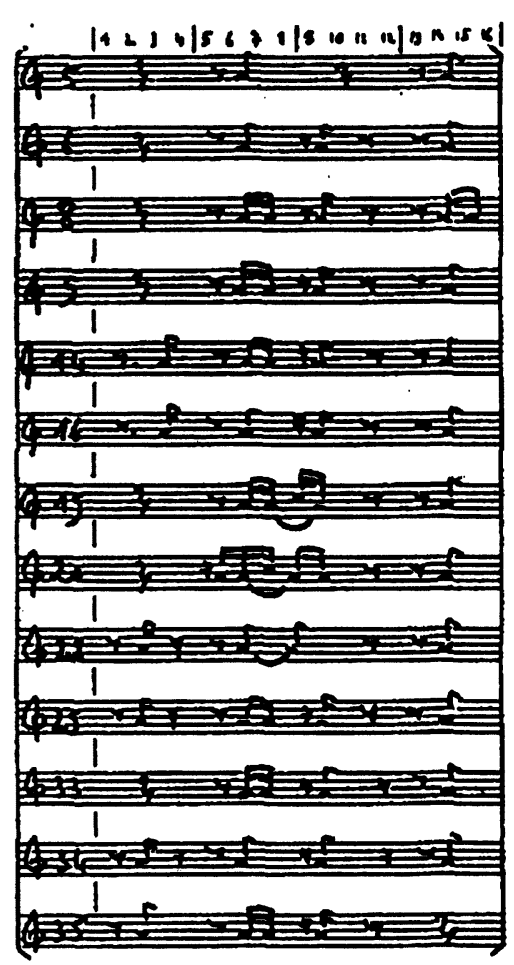

Il est démontré par l'auteur que la trompe 6 effectue ses variations selon le modèle de la trompe 1, la trompe 7, selon celui de la trompe 2, etc. En se fondant sur ce que chacun des ensembles de variations a en commun - le paradigme des paradigmes -, l'auteur peut montrer que la pièce est construite sur cinq modèles propres à chacune des cinq trompes et leurs homologues (exemple 6).

Et il peut même aller plus loin, en dressant le paradigme des paradigmes des paradigmes : le modèle de la pièce déduit des cinq modèles de l'exemple 6 (exemple 7).

Il s'agit bien sûr du modèle de base à partir duquel toute la pièce est développée par les musiciens. La même recherche de modèles, fondée sur la méthode paradigmatique, a été entreprise avec succès pour les " chants à penser » des Gbaya par Vincent Dehoux (1986). Dans ces travaux, on est passé de la description immanente de chacune des parties musicales constituantes à leur racine poïétique.

Toujours dans le champ de l'ethnomusicologie, la technique paradigmatique a débordé du côté de la sémantique externe - c'est-à-dire du renvoi au monde extérieur -, explicitement refusée par Ruwet (1972, 12-14). En lui combinant 


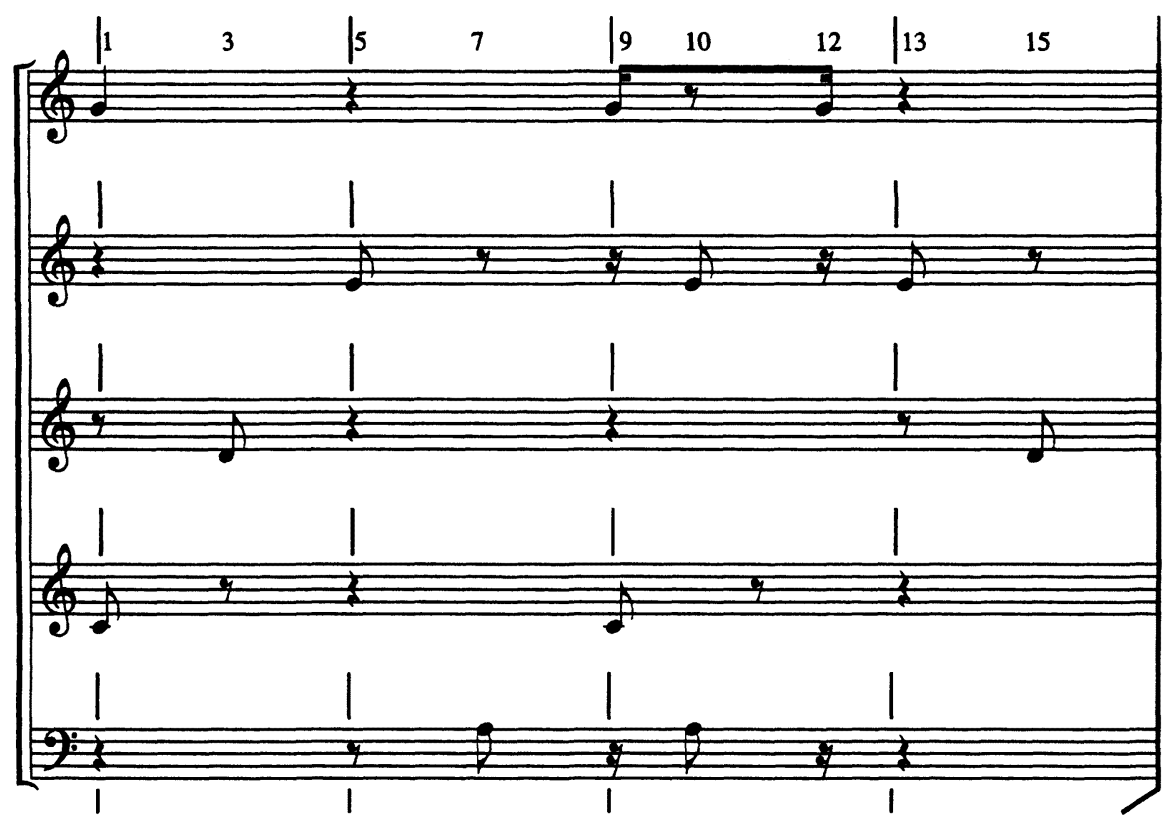

Exemple 6. Modèle pour chacune des cinq trompes de l'exemple 5 (Arom 1985, 573)

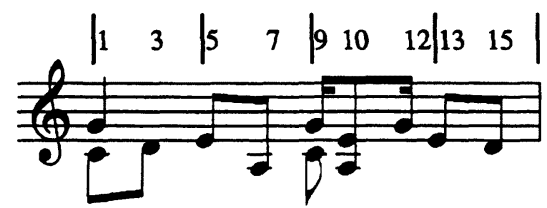

Exemple 7. Modèle de la pièce (Arom 1985, 573)

le modèle de décryptage sémantique proposé par Boilès $(1967,1973 b, 1975)$, deux membres du G.R.S.M. ont mis en rapport les données sémantiques avec les unités découpées selon les principes de la paradigmatique ruwetienne : Nicole Beaudry (1983) à propos du langage des tambours dans les cérémonies vaudou haïtiennes, et Monique Desroches $(1980 ; 1982 ; 1996,106)$ pour les indicatifs de tambour dans les cérémonies tamoul de la Martinique. Il est démontré dans son ouvrage Tambours des dieux que les unités de la colonne I dans l'exemple 8 ci-après sont associées à la déesse Mariemin. Les unités de la colonne II interviennent lors des périodes de transition de la cérémonie ou juste à la fin de celle-ci. La troisième colonne regroupe les battements utilisés pour accorder les tambours ou pour mettre en évidence la virtuosité du cheftambourineur. Les deux unités de la dernière accompagnent la transe du prêtre et sont associées au dieu Maldevilin. 


\begin{tabular}{|c|c|c|c|}
\hline 1 & $\mathrm{I}$ & $\mathbf{m}$ & $\mathrm{N}$ \\
\hline \multicolumn{4}{|l|}{ 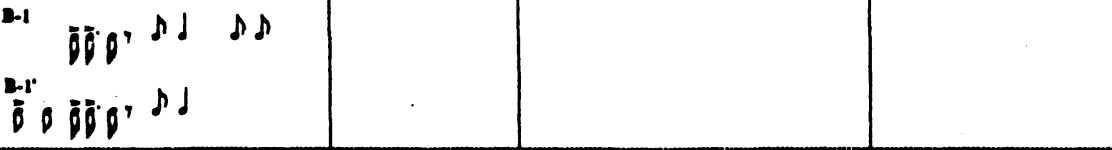 } \\
\hline & ODOBOCO & & \\
\hline \multicolumn{4}{|l|}{ 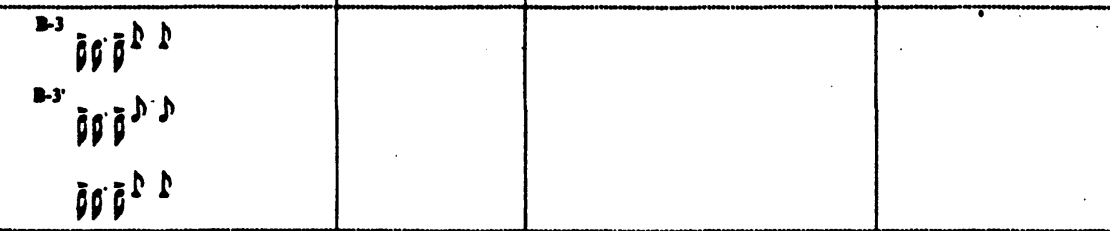 } \\
\hline & & 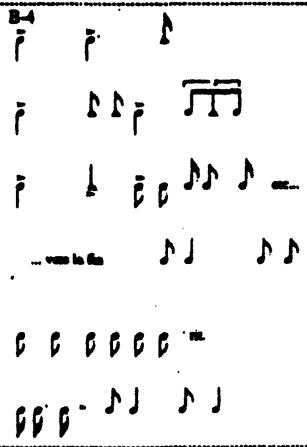 & \\
\hline & & & 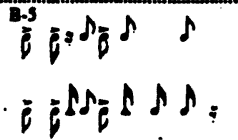 \\
\hline be ie bifesd ss & SS & & . \\
\hline
\end{tabular}

Exemple 8. Paradigme des frappes de tambour dans une cérémonie tamoule de la Martinique (Desroches 1996, 106)

Signalons encore une fort belle étude de Michael Asch (1975), fondée sur la paradigmatique, qui déborde également le niveau immanent pour démontrer que la structure formelle des chants d'un répertoire des Indiens Slavey ne coïncide pas avec les catégories vernaculaires utilisées pour désigner les différents genres.

Si l'on admet que l'analyse des musiques pop n'est pas sans lien avec l'ethnomusicologie, c'est sans doute le moment de souligner que, dans Studying Popular Music, Richard Middleton, s'appuyant sur l'article fondateur de Ruwet, en applique les principes à l'analyse de "A Foggy Day 》 de George Gershwin (Middleton 1990, 183-89). En marge de l'ethnomusicologie, on relèvera l'utilisation de la paradigmatique de Ruwet pour l'analyse des chants d'oiseaux dans les recherches d'« ornythomusicologie » du compositeur François- 
Bernard Mâche (1983) qui, par ailleurs, a consacré un article d'ensemble aux techniques d'analyse paradigmatique (Mâche 1986).

Du côté de la musique occidentale, signalons tout d'abord, dans la foulée des analyses de Ruwet consacrées au monodies médiévales, deux des articles de Lidov (1975, 35-77, 79-85) : dans le premier, où l'auteur discute l'ensemble de la méthode, il réexamine deux analyses de Ruwet; dans le second, il propose lui-même une analyse de Haut honor d'un commandement. Il utilise le format - plus que la logique - de l'analyse paradigmatique pour en ordonner les différentes unités, mais devançant un point de vue que Ruwet reprendra à son compte dans son article de 1975, il tente de montrer comment il est possible d'analyser la pièce à partir de l'idée intuitive (à partir d'une " théorie ») de ce qu'est une phrase musicale. Dans un troisième article, Lidov (1975, 87-98) propose une analyse paradigmatique de Voiles de Debussy, également selon une technique remaniée. La même année, Jean-Michel Vaccaro (1975) publiait une analyse paradigmatique de Mignonne allon voir, de Costeley, en ajoutant aux critères de répétition celui de contraste, notamment entre les densités polyphoniques, les types de contrepoint et les ambitus. Un travail de maîtrise d'Élisabeth Morin (du G.R.S.M.) a porté sur les Structures mélodiques et rythmiques d'une fugue de Bach (1979a). Prenant à la lettre une suggestion de Ruwet (1972, 107n2), elle a montré ce qu'une analyse empirique peut apporter de nouveau par rapport aux démarches qui projettent sur l'œuvre le cadre a priori, le «filtre théorique », de la conception scolastique de la fugue. La même année, elle publiait (Morin 1979b) la première tentative systématique d'utilisation du modèle paradigmatique dans le cadre d'une recherche stylistique : il s'agissait de comparer le traitement d'un même thème dans des variations de deux compositeurs différents (Byrd et Tomkins sur « John Come Kiss me Now "). Du côté de la musique classique, le thème de la Symphonie en sol mineur, K. 550 de Mozart a fait l'objet, sous la plume de Gino Stefani (1976, 37-46), d'une approche paradigmatique qui tendait à montrer comment on pouvait déborder la stricte analyse immanente pour proposer une caractérisation fonctionnelle du passage (cette approche a fait l'objet d'une discussion critique par Noske [1977]). Le même thème était abordé paradigmatiquement par Leonard Bernstein (1982, 45-49) - même si le compositeur, connaissant peu de choses à la linguistique, attribuait le modèle utilisé à Chomsky - pour en révéler une ambiguïté de structure dont le chef-d'orchestre devait tenir compte. Ce travail, qui ne doit qu'à la célébrité du compositeur de West Side Story d'avoir été publié, a fait l'objet d'une critique rigoureuse (Keiler 1978b). Ma propre analyse de l'Intermezzo, op. 119, no 3 de Brahms (Nattiez 1975a, 293-330) a mis l'accent sur l'ambiguïté des structures de cette pièce, mais en même temps, elle montrait comment chacune des deux segmentations proposées pouvait permettre, sinon de justifier, en tout cas de prévoir deux options d'interprétation de l'œuvre chez deux pianistes. Les approches de Bernstein et Nattiez on le voit, débordent du côté de la pertinence esthésique. Et Dunsby a utilisé à deux reprises (Dunsby 1982, 239-41; Dunsby et Whittall 1988, 218-23) une analyse de l'Intermezzo, op. 119, $\mathrm{n}^{\circ} 1$ de Brahms pour expliquer 
les objectifs et l'apport de l'analyse paradigmatique dans le cadre du modèle sémiologique tripartite de Molino (1975) et Nattiez (1975a, 1987) regroupant l'analyse immanente, l'analyse poïétique et l'analyse esthésique, donc débordant, encore une fois, le seul niveau structural. Annie Labussière (1992) a consacré une très belle analyse paradigmatique au solo de cor anglais de Tristan dont elle a relié les résultats aux propositions de Chailley pour l'analyse des échelles et de la mélodie. J'ai donné de l'analyse de cette pièce une autre version (Nattiez 1998), tout en m'inspirant du travail de Labussière, en la resituant dans le contexte de la sémiologie tripartite et en la combinant avec la méthode d'analyse mélodique linéaire proposée par Leonard Meyer (1973).

Pour ce qui est du XX $\mathrm{XX}^{\mathrm{e}}$ siècle, j'ai consacré au solo de basson du Sacre du printemps une analyse paradigmatique peu réussie (Nattiez 1975a, 279-85), fort justement démolie par Keiler (1981). J'ai repris ce travail dans un article récent (Nattiez 2002, 215-18; 2004, 265-69). Je me suis également attardé à la ligne mélodique de Intégrales de Varèse, combinant la paradigmatique avec la caractérisation distributionnelle (Nattiez 1975a, 290-97). J'ai consacré à Densité 21,5 du même compositeur un ample travail (1975d) qui mettait l'accent sur le déroulement syntagmatique de la pièce, mais à partir d'une définition paradigmatique des unités. Cette étude débordait, un peu timidement, sur la pertinence poïétique et esthésique des unités structurales. Marcelle Guertin (1974), membre du G.R.S.M., a consacré une belle étude à île de feu 2 de Messiaen en montrant comment l'interprète pouvait tirer partie de l'analyse paradigmatique (elle se tournait donc vers l'esthésique). C'est Gilles Naud (1975a), membre du G.R.S.M. qui, le premier, avait tenté de mettre systématiquement en relation l'analyse paradigmatique avec les pôles compositionnels et perceptifs, à propos du Nomos Alpha de Xenakis. Du côté des œuvres monodiques contemporaines, signalons encore l'étude de John MacKay (1988) sur les Sequenzas IV et $V I$ de Berio. On pourrait penser que la technique paradigmatique, fondée sur la dialectique de la répétition et de la transformation, ne peut pas être appliquée à la musique de Boulez. Jonathan Goldman (1998) a démontré le contraire en décortiquant Anthèmes, pour violon seul. En établissant des liens entre les propriétés exhibées et les concepts théoriques élaborés par Boulez, l'auteur donne à son analyse une pertinence poïétique.

Mais c'est probablement Debussy qui aura connu la plus grande « fortune paradigmatique ", sans doute parce que, avant même de publier son grand article théorique de 1966, Ruwet avait proposé, dans sa « Note sur les duplications dans l'œuvre de Claude Debussy » de 1962, une élégante analyse du Prélude de Pelléas et Mélisande. Cette dernière a fait l'objet de prolongements critiques (Nattiez et Hirbour-Paquette 1973; Nattiez 1975a, 90-93, 135-38 et 260-63) qui, en particulier, nous donnaient l'occasion d'insister sur la nécessité de procéder systématiquement à la comparaison des analyses déjà existantes : l'entreprise permettait de souligner le caractère non explicite des prédécesseurs de Ruwet dans l'analyse de ce prélude. Cette comparaison des analyses a été systématiquement pratiquée par l'essai de Morin sur la fugue en mi bémol de Bach (1979a), dans mon travail sur Densité 21,5 (1975d) et sur l'Intermezzo 
de Brahms (1975a, 327-30). Craig Ayrey (1985) s'est attaqué également à la comparaison des analyses de Bruyères de Debussy sur la base de la technique paradigmatique. On la retrouve encore dans l'ouvrage de Stefani à propos de Mozart (1976, 50-52) et dans mon analyse de Syrinx, de Debussy (Nattiez 1973d; 1975a, 338-41), où je tentais assez maladroitement, avec l'aide d'une description documentaire, de passer de l'analyse immanente à une interprétation stylistique des unités dégagées par la paradigmatique. Marcelle Guertin $(1981,1990)$ est parvenue à une mise en série, beaucoup plus réussie, des thèmes du premier livre des Préludes de Debussy, en proposant la construction de paradigmes de paradigmes qui aboutit à une caractérisation simple de leurs propriétés stylistiques. L'auteure propose également une interprétation esthésique des unités dégagées. C'est probablement, du côté de la musique occidentale, le travail le plus ample mené à ce jour dans le prolongement des propositions de Ruwet, même s'il ne porte que sur une tête d'épingle. Enfin, une analyse paradigmatique des quinze premières mesures de La cathédrale engloutie est utilisée pour démontrer la nécessité, dans le cadre de la sémiologie musicale tripartite, de distinguer entre l'analyse du niveau neutre, le poïétique et l'esthésique (Nattiez 1997a). Cette analyse sera reprise et longuement développée dans un nouveau livre en préparation.

La technique paradigmatique n'a pas toujours été utilisée pour analyser des œuvres. Elle a, méthologiquement, un caractère suffisamment général pour aider à résoudre des énigmes musicologiques plus vastes. C'est ainsi que Boilès, indépendamment de l'influence de Ruwet, l'utilise pour tenter de reconstruire les proto-mélodies d'un répertoire (1973a), ou pour déterminer son degré d'acculturation (1982). La méthode de Ruwet conduit également à expliciter les relations de transformations entre unités. Cet aspect a fait l'objet de recherches spécifiques dans Nattiez (1975a), Brediceanu (1975), Cazimir (1975), Levy (1975) et Naud (1975b).

On peut tirer deux grandes conclusions de ce survol bibliographique et historique :

1) L'analyse paradigmatique est assez vite sortie de la perspective structuraliste qui était celle de Ruwet dans les années 1960 pour déborder du côté des stratégies compositionnelles et perceptives, des associations sémantiques et passer de l'étude de la spécificité d'une œuvre dans son unicité à une perspective stylistique plus large. Il n'y a rien là d'étonnant, car au structuralisme pur et dur des années 1960 a succédé, en linguistique, en théorie littéraire comme en musicologie, une période poststructuraliste dans laquelle nous vivons toujours et qui mérite son nom, non pour désigner les dérives de la déconstruction, mais pour rappeler que les recherches nouvelles se doivent d'intégrer, y compris de façon critique, les acquis des recherches immanentes.

2) La méthode s'est surtout intéressée à des corpus essentiellement monodiques et à des périodes " intermédiaires " de l'histoire de la musique (musique médiévale, Debussy), à des compositeurs du $\mathrm{XX}^{\mathrm{e}}$ siècle (Varèse, Xenakis, Berio) ou aux musiques extra-européennes, là où il était le plus légitime qu'une méthode explicite $d$ 'analyse fonctionne en feignant de mettre entre parenthèses 
les intuitions ordinaires du musicien et le savoir acquis des théoriciens et des musicologues.

À en juger par la richesse de cette postérité musicologique, Ruwet avait certainement vu juste, dès 1966, lorsqu'il déclarait que « bien des choses ici énoncées seront à reprendre et à élaborer » $(1972,133)$. Ce sont les principaux amendements apportés à la théorie et au modèle initial que je voudrais examiner maintenant.

\section{Modifications apportées au modèle de Ruwet}

$\mathrm{Au}$ fur et à mesure que les propositions de Ruwet ont fait l'objet d'applications concrètes, des changements techniques ont été apportés aux règles explicites d'analyse proposées dans l'article de 1966. Ruwet avait donné la longueur comme critère de la répartition hiérarchique des unités. L'expérience a prouvé qu'il y avait là un parti pris de régularité et de symétrie qui perdait sa pertinence dès que l'on sortait du style classique. Il est ainsi apparu qu'il n'y avait pas de limites a priori au nombre de critères pouvant intervenir dans l'association paradigmatique, comme l'ont montré Arom $(1969,205-6)$ et Nattiez (1975d) : au critère dominant de la répétition peuvent s'ajouter aussi bien la distribution des intervalles, le rôle des intensités, la combinaison des points d'attaque de l'analyse, etc. Cette extension de la critériologie n'a fait que confirmer une des observations les plus judicieuses de Ruwet : « Il est impossible de représenter la structure d'une pièce musicale par un schéma unique » $(1972,134)$. S'il avait eu tendance à considérer seulement comme des indices les critères de contrastes, de pause, de phrasé, voire de cadence $(1972,106)$, parce qu'il attribuait au critère de répétition un poids hiérarchique dominant - la répétition est le " critère principal de division " $(1972,111)$ - , ces critères ont vite été réintroduits par tous dans l'analyse comme correspondant à notre intuition musicale immédiate. Mais on observera - et ici, le principe de la séparation des paramètres reste décisif - que le phrasé ne correspond pas nécessairement à la division paradigmatique qu'impose le contour mélodique. Il peut donc être capital, à un stade provisoire de l'analyse, de les distinguer (Nattiez 1975d).

Mais la critique la plus violente contre les propositions initiales de Ruwet est venue... de Ruwet lui-même qui, dans son article de 1975, sous l'influence de la pensée chomskyenne, a voulu tourner le dos au modèle harrissien qui l'avait inspiré dans l'explicitation des procédures d'analyse, en redonnant à l'intuition - sous le nom de "théorie " - la place que son article de 1966 semblait lui avoir contestée. À cet égard, son analyse du découpage des trois premières mesures du Concerto pour piano, K. 271 de Mozart (Ruwet 1975, 30) est tout à fait éloquente, puisqu'il réintroduit les critères de contraste, les pauses et la dimension harmonique. Aujourd'hui, l'émotivité du clivage épistémologique entre " empiristes " harrissiens et " théoristes " chomskyens s'étant estompée, il semble qu'il n'y a plus de difficultés à tenir compte des intuitions tonales dans une démarche analytique fondée sur l'explicitation. C'est sans doute ce qui explique que si l'article de 1975 a été salué comme une saine auto-critique de l'auteur (Powers 1980), en revanche, comme le souligne 
judicieusement Everist $(1987,6)$, c'est l'article de 1966 qui a exercé et exerce encore la plus grande influence empirique.

Mais je voudrais souligner un point important. Si plusieurs analyses d'œuvres occidentales ont pu être menées correctement en mettant entre parenthèses des critères immédiatement fournis par le sentiment tonal, cela prouve que, pour un même passage musical, on peut faire appel à différents critères qui se révèlent convergents. Lorsque, en 1966, Ruwet ne retenait que les critères de répétition/transformation, il proposait une méthode qui transcendait la spécificité de chaque système musical particulier et touchait ainsi, comme on l'a dit, aux universaux de la musique. Les critères de pause et de contraste semblent de même nature. Comme c'est le cas dans l'analyse que Ruwet propose du Prélude de Pelléas, cela expliquerait qu'on puisse faire l'économie, pour rendre compte de la segmentation d'une pièce, de son organisation tonale et harmonique, qui, elle, est propre à la culture musicale occidentale. Ce même phénomène nous semble confirmé par la démarche de Lerdahl et Jackendoff qui décrivent la structure métrico-rythmique d'une œuvre tonale sans faire intervenir dans leurs règles notre connaissance du fonctionnement de la tonalité (1983a, 13-104) et les conduit à distinguer, parmi leurs règles, celles qui seraient d'application universelle (1983a, 345-52).

La distinction entre critères universels et critères culturellement spécifiques est d'une importance théorique considérable. Du point de vue d'une ethnomusicologie générale qui, comme on commence à le faire, inclurait... la musique tonale! Du point de vue des approches cognitives, puisqu'il leur importe de savoir si telle règle, tel processus musical dépend des « structures universelles de l'esprit humain " ou de l'apprentissage d'un système musical particulier. La nature explicite des règles de Ruwet aura obligé à poser le problème.

\section{Le code et la table rase}

Il y avait, à coup sûr, dans les propositions de Ruwet en 1966, une fascination pour l'idéal de la " table rase " que mes tout premiers travaux, insistant sur la nécessité d'une analyse " neutre » de la musique, selon les termes de Molino (1975), ont peut-être contribué à accentuer ${ }^{9}$. Cet aspect de la méthode a soulevé suffisamment de débats pour que Ruwet le dénonce avec véhémence dans son article de 1975. Mais une relecture attentive des articles de 1962 et 1966 conduit à penser que la position de Ruwet, à l'époque, était déjà beaucoup plus nuancée. Il convient de s'attarder en particulier à l'usage qu'il fait du mot " code ".

Ruwet $(1972,104)$ constate d'abord que la distinction entre " message » et " code ", si fréquente dans la linguistique et la sémiologie de l'époque, n'a pas été thématisée dans la recherche musicologique et que, dans le mouvement analytique qui permet de "remonter " du message au code, les critères et les procédures n'ont jamais fait l'objet d'élaboration explicite. Le code, c'est donc

9Dans Musicologie générale et sémiologie, j'insiste beaucoup, pour éviter les ambiguîtés, sur la nécessité de parler d'« analyse du niveau neutre " (Nattiez 1987, 34, 62n12). Je parle aussi volontiers de " niveau immanent ". 
l'aboutissement d'une procédure analytique qui découvre les structures de la pièce ou du corpus dans une situation d'ignorance absolue : « La démarche [du message au code] s'impose chaque fois que, s'agissant d'une langue inconnue, d'un mythe ou d'une musique exotique, etc., le message est le seul donné " (Ruwet 1972, 100). Et Ruwet $(1972,107)$ de souligner l'importance des procédures de découverte dans l'étude des musiques non européennes.

Mais on ne perdra rien ici à enregistrer l'ambiguïté du mot " code ", surtout utilisé au singulier. Car de quel code s'agit-il? La structure formelle de la pièce, les relations entre ses différents paramètres, le système scalaire (pentatonique, modal, tonal, chromatique)? Tout cela à la fois, probablement. Utilisé dans la perspective structuraliste de l'époque, le mot " code » désignait les éléments qui permettent au destinataire de comprendre (de " décoder ") ce qu'a voulu transmettre l'émetteur. Or, il est probable que, au sein de cette entité « code ", il soit nécessaire de distinguer entre les aspects effectivement communs au compositeur (ou au producteur de musique) et à l'auditeur (les échelles sont souvent de ceux-là), et ceux qui échappent à la pratique codifiée d'une époque. C'est une raison de plus, à mon sens, pour entreprendre des descriptions immanentes empiriques particulières afin de mettre en évidence la spécificité d'une pièce ou d'un corpus par rapport à la pratique d'une époque donnée.

Mais en même temps que Ruwet utilise la distinction message / code dans un contexte où l'on est dans l'ignorance du " code ", il reconnaît que nous pouvons disposer d'indices sur le contenu de ce code $(1972,102)$, comme la description des instruments, les renseignements sur la manière d'en jouer, les données sur les conditions d'exécution, des commentaires divers, les titres. Avec ces exemples, Ruwet semble montrer qu'en élaborant son modèle d'analyse, il a surtout en tête les musiques qui sont étrangères à notre culture, dans le temps ou dans l'espace : les monodies médiévales ou les musiques de tradition orale. Mais on ne peut oublier que c'est à l'ensemble des modèles analytiques que Ruwet reproche l'absence de procédures explicites.

Dès lors, la méthodologie de Ruwet allait s'appliquer dans deux contextes de recherche :

1) les corpus musicaux dont on ne sait à peu près rien, ou dont on croit ne rien savoir : ce qui explique à la fois le succès de son modèle auprès des ethnomusicologues et son propre intérêt pour les monodies médiévales;

2) les aspects de musiques plus familières, dont la prise en charge par les théories traditionnelles est soit inexistante soit insatisfaisante. Si, en 1962, Ruwet analyse le Prélude de Pelléas, c'est parce que la notion de duplication proposée par Schaeffner correspond à une intuition juste (fondée sur une observation attentive), mais qui n'a pas fait l'objet d'une investigation systématique permettant d'en préciser les caractéristiques structurelles et les fonctions. De la même façon, on constate que les études paradigmatiques appliquées à la musique occidentale ont porté non pas sur la totalité des paramètres des œuvres, mais sur les aspects les moins codés du langage musical occidental : les structures mélodiques et rythmiques. Enfin, comme on l'a vu, elles ont surtout choisi, comme corpus, des périodes intermédiaires de l'his- 
toire de la musique, pré- ou post-tonales, qui ne relèvent pas du fonctionnement tonal au sens strict.

Le problème de l'article de 1966 venait donc de ce que, tout en reconnaissant explicitement l'existence d'intuitions sur le code, la liste des procédures explicites d'analyse semblait relever, elle, de l' " objectivisme assez primaire » dénoncé quelques pages plus haut (Ruwet 1972, 102). En un mot, la liste ne semblait pas encourager l'intégration des données fournies, soit par notre acculturation à notre système musical lorsqu'il s'agit de musiques tonales, soit par les connaissances musicologiques. Cette ambiguiité a été relevée par les critiques les plus attentifs (Lidov 1975, 57). Powers en particulier $(1980,11)$ a reconnu dans l'article de 1966 l'embryon des idées que Ruwet allait développer dans son texte de 1975 , à savoir la place pleinement reconnue à l'intuition.

Qu'en est-il aujourd'hui? S'il n'y a aucune raison de principe d'exclure a priori les « intuitions sur le code » ou, plus largement, les connaissances acquises, il me parait capital de souligner ce que je crois être l'apport essentiel des propositions de Ruwet : trop de théories et d'analyses musicales ont été élaborées sans le souci d'expliciter et de préciser les critères qui présidaient à leur construction pour qu'on n'aborde pas des aspects fondamentaux, « incertains " ou ignorés, de " nos " musiques, comme si nous avions affaire à des musiques de tradition orale, comme si elles nous étaient étrangères, comme si les théories les mieux assises risquaient d'en occulter des propriétés spécifiques et importantes. Certes, les théories transmises par l'enseignement académique reflètent une " pratique commune " dont on peut difficilement nier le rôle dans la création et la perception des œuvres; mais à partir du moment où on fait l'hypothèse que les musiques, en raison de leur complexité paramétrique, ne sont pas nécessairement perçues telles qu'elles ont été conçues, il redevient urgent de se doter d'instruments empiriques de description, et de faire la différence entre les normes pédagogiques des traités et ce que les compositeurs ont effectivement faits, ce qui, au-delà, devrait permettre de s'interroger sur les stratégies compositionnelles qu'ils ont suivies et les stratégies perceptives auxquelles ces œuvres donnent lieu.

À partir de là, bien sûr, une question fondamentale se pose : la méthode de Ruwet proposée en 1966 est-elle suffisante? L'auteur n'a jamais prétendu que ses propositions permettaient de décrire la totalité de la substance musicale d'une œuvre. Ses procédures explicites ne concernaient que les mélodies, même si, l'analyse de Pelléas publiée quatre ans plus tôt démontrait qu'elles pouvaient être étendues au problème classique de l'analyse empirique de la forme. Chez Ruwet, il n'y a pas de théorie de la relation entre le rythme et le mètre, mais une méthode fondamentale pour opérer et décrire la segmentation à l'intérieur de laquelle les autres paramètres peuvent venir prendre place, comme il l'a montré pour le paramètre harmonique (Ruwet 1967a).

C'est d'ailleurs du côté de l'harmonie que des avenues, s'inspirant de la technique paradigmatique, sont prometteuses. Dans la mesure où la structure harmonique d'une œuvre peut être ramenée à une succession de fonctions et de chiffrages, il est aisé d'appliquer cette méthode à l'analyse de séquences 
abstraites d'enchaînements harmoniques. Même si, malheureusement, cette direction n'a pas fait l'objet d'intenses recherches - mais on relève les contributions de Lidov $(1978,48)$ et de Sadai $(1986)$ - , on voit bien comment l'analyse empirique des successions harmoniques chez un compositeur donné (Baroni, Dalmonte et Jacoboni 1999, chap. 11) pourrait être comparée aux schémas syntagmatiques généraux, habituellement enseignés dans les traités (R. F. Goldman 1965), et montrer ainsi comment l'harmonie tonale a évolué de Bach à Wagner.

L'orientation des recherches analytiques récentes montre clairement comment les propositions de Ruwet peuvent s'intégrer aux travaux en cours. Aussi bien $A$ Generative Theory of Tonal Music (Lerdahl et Jackendoff 1983a) que Les fondements de la musique tonale (C. Deliège 1984) montrent qu'il est nécessaire aujourd'hui de juxtaposer ou de combiner l'étude de structures taxinomiques et celle des prolongations, inaugurée par Schenker (et absente des travaux de Ruwet comme de tous les travaux analytiques de langues françaises et italiennes de l'époque) et poursuivies par Meyer et Narmour. Historiquement, c'est sans doute à Meyer qu'on doit d'avoir, le premier, combiné ces deux approches à propos d'un scherzo de Beethoven (Meyer 1973, 81-88). L'auteur de ces lignes a fourni une première démonstration de l'intégration de la paradigmatique de Ruwet et de l'analyse prolongationnelle de Meyer (souvent appelée « implicationnelle-réalisationnelle ») à propos du solo de cor anglais de Tristan (Nattiez 1998).

Tout l'édifice analytique de Lerdahl et Jackendoff repose sur une taxinomie première, fondée, dans leur théorie, sur le rythme et le mètre. Même s'ils ne le disent pas, leur entreprise est analogue dans son fonctionnement à celle de Ruwet puisque, à partir d'instruments méthodologiques différents, ils découpent des unités mélodico-rythmiques de nature identique. Lorsque les règles explicites de Lerdahl et Jackendoff et de Ruwet, et les résultats auxquels conduisent les deux méthodologies auront été comparés, lorsque les segmentations paradigmatiques et transformationnelles auront été intégrées, avec l'analyse des paramètres dont Ruwet ne traite pas, dans un modèle global où la dimension prolongationnelle ${ }^{10}$ est d'ores et déjà présente, alors son apport fondamental à l'analyse musicale contemporaine aura trouvé sa juste place (Nattiez 1992a).

\section{LES GRAMMAIRES MUSICALES}

L'œuvre et les méthodes de Noam Chomsky, inventeur de la grammaire générative, ont exercé une influence à la fois décisive et variée sur l'analyse musicale. Décisive, parce que le projet initial de Chomsky dans Syntactic Structures (1957), répondait aux aspirations épistémologiques des années 1960 : la précision de la

$10 \mathrm{C}$ 'est sans doute parce que cette dimension prolongationnelle n'était pas prise en charge par la linguistique structurale qu'elle n'a pas inspiré de recherches en ce sens dans l'application des modèles linguistiques à l'analyse musicale. Dans l'histoire de l'analyse au $\mathrm{XX}^{\mathrm{e}}$ siècle, la prolongation vient d'autres sources : du contrepoint de Fux chez Schenker, de la psychologie de la Gestalt chez Meyer. $\grave{A}$ noter que si les théories linguistiques de Gustave Guillaume, inventeur d'une « psycho-mécanique du langage " occultée durant la période structuraliste, avaient été davantage remarquées, la situation aurait peut-être été différente. Mais on ne refait pas l'histoire... 
description et la vérifiabilité de la construction. Il s'agissait en effet de construire un algorithme de règles explicites capables d'engendrer, par un nombre fini de règles, l'infinité des phrases acceptables dans une langue, tout en leur assignant une description structurale. Mais la pensée chomskyenne comprend aussi des aspects philosophiques, psychologiques et épistémologiques. Ils concernent les universaux du langage, une théorie de compétence linguistique qui contribuera au développement plus récent des recherches dites cognitives, sans parler du cadre épistémologique par lequel Chomsky entend se démarquer de l'empirisme de la linguistique américaine, de Bloomfield à Harris. Enfin, la pratique et la théorie de la grammaire générative ne sont pas restées identiques depuis sa naissance. L'important ouvrage de 1965, Aspects of the Theory of Syntax, bientôt désigné par l'étiquette de " théorie standard ", introduit les notions de "structure de surface " et de " structure profonde ", et met l'accent moins sur la génération d'un nombre infini de phrases que sur la fonction descriptive de l'ensemble des règles : «Une grammaire parfaitement adéquate doit assigner à chaque élément d'un ensemble infini de phrases une description structurale indiquant comment cette phrase est comprise par le locuteur-auditeur idéal » (Chomsky 1965, 4-5). On ne s'étonnera donc pas que les musicologues qui se sont référés à Chomsky aient pu s'inspirer d'aspects ou d'idées diverses qui font que le " générativisme musical » se présente sous des aspects multiples.

\section{Schenker et le générativisme}

Très tôt (Forte 1959), les théoriciens qui ont adopté le modèle de Schenker pour l'analyse harmonique ont été frappés par l'analogie remarquable qu'il présente avec celui de la théorie standard de Chomsky. Le linguiste propose de générer une structure de surface à partir d'une structure profonde par l'intermédiaire de transformations; Schenker identifie une base sous-jacente (Hintergrund), une base médiane (Mittelgrund) et une base génératrice de la surface (Vordergrund). L'analogie n'a pas manqué de susciter des travaux visant à utiliser le modèle chomskyen pour présenter une analyse schenkerienne ou discuter de méthodes d'analyse musicale, notamment ceux de Kassler (1975, 1977), Keiler (1978a, $1978 b, 1981$ ) et Smoliar (1980). Le psychologue cognitif de la musique John Sloboda consacre une section de son ouvrage The Musical Mind $(1985,11-17)$ à une comparaison de Chomsky et Schenker. Blacking s'est emparé, de manière quelque peu métaphorique, des notions de structures profondes et de structures de surface pour désigner les rapports entre le soubassement culturel et les productions musicales (Blacking 1971a, 1971b), peut-être parce que, dans sa théorie générale (1973), il espère " générer » le musical à partir du culturel.

À la différence de l'approche paradigmatique, qu'on qualifie fréquemment de taxinomique ou de classificatoire, la perspective chomskyenne est hypothético-déductive. Elle propose de partir de notre intuition sur la structure du domaine étudié, puis on établit des règles pour en rendre compte et on les modifie s'il s'avère que leurs conséquences sont inacceptables. Un certain nombre de travaux ont donc utilisé le modèle chomskyen pour tester la pertinence d'une théorie classique déjà constituée. C'est la direction théorique que Ruwet proposait en 1975. Dans le même esprit, on peut citer tout particulière- 
ment le travail empirique de Rothgeb (1968) qui part des traités de basse chiffrée et examine quelles règles manquent à la théorie pour qu'il soit possible de générer mécaniquement le résultat musical escompté.

\section{La grammaire générative comme outil de la stylistique}

Selon les principes initiaux de Chomsky, une grammaire générative comprend deux composantes essentielles :

1) Un ensemble de règles qui décrivent les liens et la hiérarchie existant entre les « constituants immédiats " d'une phrase (Chomsky 1957, chap. 4.1.) :

(1) Phrase $\rightarrow S N+S V$

(2) $S N \rightarrow A r t+N$

(3) $S V \rightarrow$ Verbe $+S N$

(4) $\mathrm{Art} \rightarrow$ The

(5) $N \rightarrow$ man, ball, etc.

(6) Verbe $\rightarrow$ hit, took, etc.

Ces règles sont dites de "réécriture ", car elles vont de l'unité la plus vaste (toujours la phrase) à ses premiers constituants, ici le syntagme nominal et le syntagme verbal. Le syntagme nominal se réécrit à son tour « article » + «nom », puis on en arrive aux " éléments terminaux » : the, man, hit, took, etc.

2) À cet ensemble de règles correspond un « indicateur syntagmatique », une structure arborescente qui résulte de l'application de ces règles et montre la structure sous-jacente et hiérarchique de la phrase. L'exemple 9 montre une des dérivations possibles des six règles proposées.

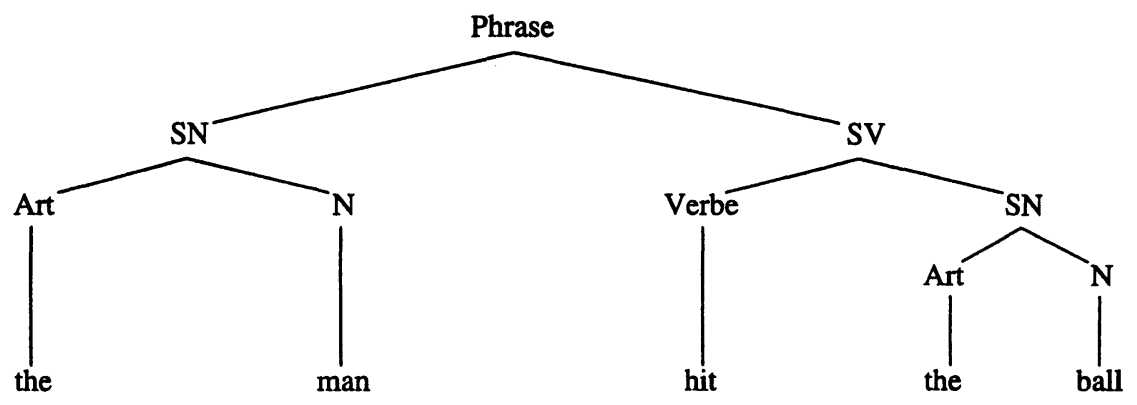

Exemple 9. Indicateur syntagmatique d'une phrase anglaise (Chomsky 1957, chap. 4.1)

Inutile de dire que la linguistique générative n'a jamais réussi à proposer la description complète d'une langue donnée, et un auteur comme Maurice Gross, d'orientation harrissienne, a pu montrer, dans deux monumentales analyses du verbe et du nom en français $(1968,1977)$ et dans un texte théorique de grande envergure (1979), que l'école chomskyenne a superbement ignoré, pourquoi il ne pouvait pas en aller autrement : "If we compare, two-by-two, the syntactic 
properties of any two lexical items, it is observed that no two lexical items have identical syntactic properties " (Gross 1979, 860). Autrement dit, les règles transformationnelles - qui sont une des composantes de la grammaire générative - seront, en réalité, en nombre infini. On peut soupçonner que c'est, non sous l'influence de Gross, mais devant la démultiplication du nombre de règles transformationnelles nécessaires à la description d'une langue, que Chomsky a progressivement donné comme objectif à la grammaire générative, non de fournir un ensemble fini de règles capables de générer un nombre infini de phrases, mais d'assigner aux phrases une description structurale.

La situation est quelque peu différente en musique, car, prenant au sérieux la possibilité de décrire le style d'un genre ou d'un compositeur, les musicologues, le plus souvent, ont travaillé sur des corpus à la fois simples et bien délimités. De la même façon que, étant donné la nature même de la musique comme forme symbolique, le structuralisme était sans doute plus approprié pour décrire la musique qu'une œuvre littéraire ou une langue, je me permettrai de suggérer que le générativisme strict de Chomsky (la construction d'un ensemble de règles générant et décrivant hiérarchiquement un ensemble de phrases plus nombreuses que le corpus initial d'analyse) a eu plus de succès en musicologie qu'en linguistique.

Dans le domaine musical, l'outil génératif s'adapte particulièrement bien à la description explicite d'un style, à la condition que le corpus soit suffisamment simple pour faire l'objet d'un ensemble fini de règles qui permettent la génération de "faux " reconnus par des experts comme appartenant au même ensemble, tout en assignant aux productions musicales une description syntagmatique qui en explicite l'organisation immanente. Personne n'a encore proposé de grammaire générative du style de Wagner...

Aussi, la méthode générative a-t-elle été surtout utilisée en ethnomusicologie, dans les études de jazz et de musique pop, parce qu'elles ont souvent affaire à des monodies. Les travaux se réclamant de la grammaire générative, dont certains programmatiques ${ }^{11}$, sont nombreux ${ }^{12}$, et chacun d'entre eux mériterait une discussion méthodologique attentive et détaillée. Dans le cadre limité de cet article, on retiendra, à titre de modèle pédagogique, la grammaire de srepegan javanais élaborée par Judith et Alton Becker (1979), suffisamment

11 Avec l'expansion de l'usage de l'ordinateur dans la recherche scientifique, la frontière peut être difficile à établir entre grammaires génératives musicales et utilisation de l'ordinateur dans la solution des problèmes musicologiques. Nous ne considérons pas ici les grammaires dites de reconnaissance et celles qu'on utilise pour la composition musicale. Deux publications permettent de faire le point sur les grammaires musicales de toute nature : le Computer Music Journal 9, $n^{\circ} 2$ (Collectif 1980) et les actes de l'important colloque tenu à Modène en 1980 (Baroni et Callegari 1984). Nous excluons de cette liste les grammaires génératives utilisées pour la composition. On trouvera d'autres références dans Baroni, Dalmonte et Jacoboni $(1999,2 \ln 8)$.

12 Voir Baroni et Jacoboni (1976), Baroni et al. (1984), Boilès (1967), Brediceanu (1975), Camilleri (1984), Chenoweth et Bee (1971), Cooper (1973), Giomi et Ligabue (1988), Herndon (1975), Hughes (1991), Johnson-Laird (1991), Kippen (1987), Kippen et Bel (1992), Laske (1972-73), Lidov et Gabura (1973), Lindblom et Sundberg (1970, 1972), Middleton (1990, 189-214), Pelinski (1981b), Perlman et Greenblatt (1981), Sapir (1969), Steedman (1984), Sundberg et Lindblom $(1972,1976)$, Wenk (1988) et Winograd (1968). 
efficace pour qu'il soit possible de fabriquer soi-même de nouvelles pièces à partir des règles proposées (exemple 10). De plus, cet article montre quelle utilisation le musicologue peut faire de certains symboles fort simples propres à donner une représentation explicite des répétitions, des effacements, des enchâssements de structures dans des contextes bien définis.
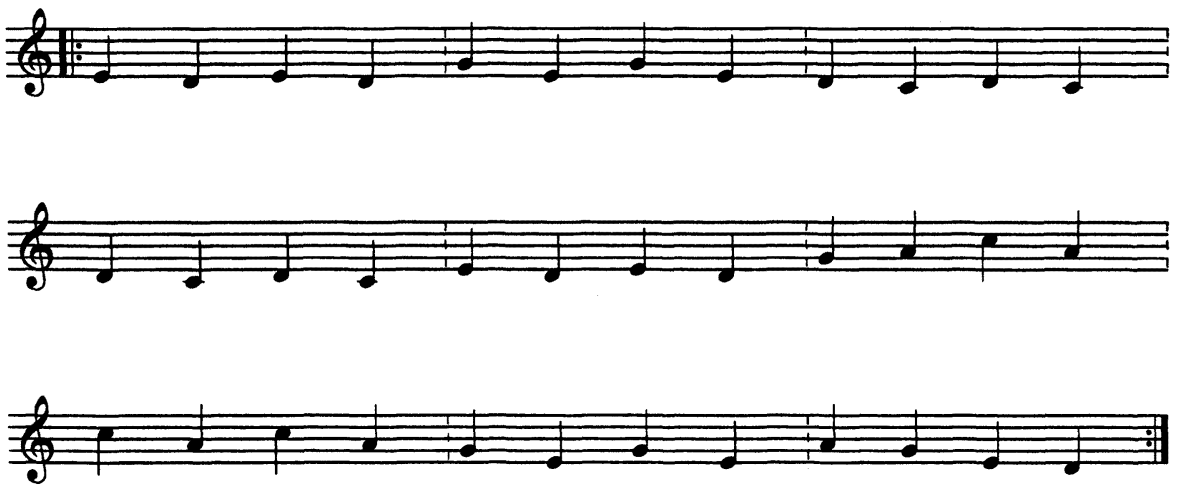

Exemple 10. Exemple de srepegan, slendro pathet manyura

Soulignons que, pour pouvoir proposer une grammaire générative de l'ensemble du corpus auquel cette pièce appartient, il faut d'abord en établir une segmentation qui, ici, repose sur des catégories émiques. Chacune des trois lignes se termine par un coup de gong et est dénommée gongan. Chaque gongan est subdivisé en unités de quatre mesures qui semblent musicalement distinctes pour nous, mais qui, pour les autochtones, sont apparentées non selon les hauteurs, mais selon les contours (exemple 11).

Ainsi, le premier gongan de l'exemple 10 est-il fait de trois unités : 1, 1, 1; le second comprend $1,1,2$ et le troisième : 1, 1, 3. Après analyse du corpus, les auteurs proposent une grammaire générative (exemple 12).

La règle 1 se lit comme suit : un srepegan se compose d'un $G_{i}$, d'un $G_{j}$ et d'un $G_{i}$. Les $i$ distinguent les gongans du début et de la fin de la pièce (signalés par les \#), le $G_{j}$ désigne le gongan situé à l'intérieur de la pièce et non à ses extrémités. La seconde règle prescrit qu'un gongan de début ou de fin $\left(G_{i}\right)$ se réécrit : $a$, optionnel (le $a$ est entre parenthèses), suivi du contour 1 et suivi de $b$. Les règles suivantes vont réécrire $a$ et $b$. (On sait déjà que $l$ est un contour intonatif spécifique [exemple 11]). Quant au gongan central, il a toujours une structure immuable $(1+1+2)$. La règle 4 signifie que $a$ se réécrit ou $G_{i}$ ou $l$ ou les deux. La règle 5 est la plus complexe et la plus riche. Elle indique que $b$ peut se réécrire 1 ou 3 , ou 4 et 6 , ou 2 (l'option est indiquée par des accolades). Chacune des options est spécifiée selon le contexte, indiqué par la barre oblique $/$. Selon la première sous-règle, pour que $b$ se réécrive 1 , là où il y a un trait souscrit, il faut qu'il apparaisse dans un gongan de début et qu'il soit précédé des unités $1+1$. Pour que $b$ soit réécrit 2 , il doit être dans un gongan de la fin de la pièce. Il est réécrit 4 s'il apparait dans une unité dominée par $a$ (cela 


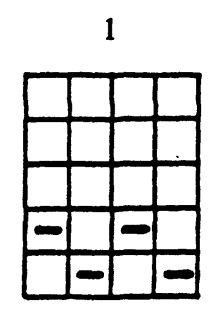

3

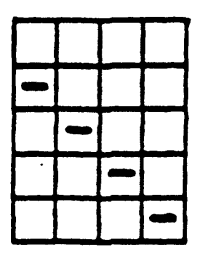

5

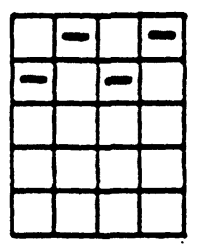

2

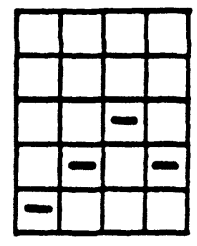

4

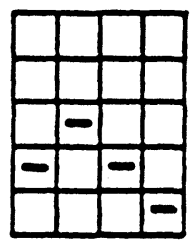

6

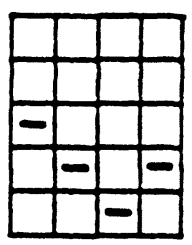

Exemple 11. Inventaire émique des contours utilisés dans le genre srepegan

1. Srepegan $\# G_{i}+G_{j}+G_{i} \#$

2. $G_{i} \quad[(a)+1+b]$

3. $\mathrm{G}_{\mathrm{j}} \quad[1+1+2]$

4. a $\left(G_{i} / 1\right)$

5.

b $\left\{\begin{array}{l}1 \\ 3\end{array}\right\} / \mathrm{G}_{\mathrm{i}}[1+1+\ldots]$

$$
\left\{\begin{array}{l}
4 \\
6
\end{array}\right\} / \#_{a}\left[1+G_{j}+\right]_{a}[1+]_{a}+G_{i} \#
$$

2

Exemple 12. Grammaire générative du genre srepegan 
suppose qu'on a d'abord appliqué la règle 4 ), immédiatement après le début de la pièce. Il se réécrit $6 \mathrm{~s}$ 'il apparaît dans une unité dominée par $a$, précédée d'un gongan interne et suivi d'un gongan de fin. Il se réécrit 2 dans tous les autres cas. $\mathrm{D}$ 'autres règles viennent spécifier quelles sont les notes réelles qui réalisent les contours 1 à 7 selon les contextes. Le lecteur peut maintenant s'entraîner à dériver des structures de srepegan javanais... Il découvrira qu'il $y$ en a une infinité.

Ce qui est remarquable dans cette grammaire, sur les plans à la fois pédagogique et épistémologique, c'est que nous sommes bien en présence d'un système de règles explicites dans lequel tous les éléments variables sont définis par leur contexte. Nous sommes bien en présence d'une description rigoureuse et immanente du style d'un genre particulier. On ne saurait prétendre que ce système de règles correspond aussi aux stratégies poïétiques des musiciens indonésiens (" génératif » ne signifie pas " compositionnel ») ni aux stratégies esthésiques de leurs auditeurs : il décrit les contraintes syntagmatiques des éléments constitutifs du corpus, ce qui est déjà beaucoup.

\section{Un modèle génératif de la compétence perceptive tonale}

Plus Chomsky a évolué, plus il a insisté sur la pertinence psychologique des grammaires génératives, d'où le lien qui s'est établi entre le chomskysme et la psychologie cognitive. C'est parce qu'ils se sont donné comme objectifs de fournir les règles générales qui commandent la perception de la musique, que Lerdahl et Jackendoff ont intitulé leur livre : A Generative Theory of Tonal Music (1983a). Comme l'indiquent explicitement les auteurs au début de leur travail, « we must mention some common misconceptions about generativetransformational grammar. The early work in the field, such as Chomsky 1957 and Lees 1960, took as its goal the description of "all and only" the sentences of a language, and many were led to think of a generative grammar as an algorithm to manufacture grammatical sentences. Under this interpretation, a musical grammar should be an algorithm that composes pieces of music $»$. Et ici les auteurs renvoient à quelques-uns des auteurs de grammaire générative que j'ai cités plus haut. Ils continuent ainsi :

There are three errors in this view. First, the sense of "generate" in the term "generative grammar" is not that of an electrical generator that produces electricity, but the mathematical sense, in which it means to describe a (usually infinite) set by finite formal means. Second, it was pointed out by Chomsky and Miller (1963), and it has been un unquestioned assumportion of actual research in linguistics, that what is really of interest in a generative grammar is the structure it assigns to sentences, not which strings of words are or are not grammatical sentences. The same holds for our theory of music. It is not intended to enumerate what pieces are possible, but to specify the structural description for any tonal piece; that is, the structure that the experienced listener infers in his hearing of the piece. A third error in the conception of a generative grammar as a sentence-sweping device is not evident from passing acquaintance with the early works of the generative school, but emerges as a prominent theme of Chomsky 1965, Lenneberg 1967, and subsequent work. 
Linguistic theory is not simply concerned with the analysis of a set of sentences; rather it considers itself a branch of psychology, concerned with making empirically verifiable claims about one complex aspect of human life: language. Similarly, our ultimate goal is an understanding of musical cognition, a psychological phenomenon. (1983a, 6)

J'ai tenu à citer au complet ce long passage, afin que le lecteur intéressé par les applications de la linguistique à la compréhension du phénomène musical puisse se retrouver dans les différents usages qui sont faits, et en linguistique et en musicologie, du mot " génératif ". La mise au point initiale de cette section sur la diversité et l'évolution de la théorie chomskyenne aura permis de comprendre pourquoi la perspective "générative " de Lerdahl et Jackendoff est complètement différente, dans ses objectifs et ses méthodes, de celle des auteurs de grammaires stylistiques cités précédemment, même si le terme d'" erreur " de conception utilisé ici est probablement exagéré. Si on peut admettre qu'il soit légitime d'utiliser le concept de générativisme pour qualifier une théorie de la perception musicale qui s'inspire d'un des aspects de la théorie chomskyenne, il n'y a pas de raison d'exclure du paradigme chomskyen, au sens large, la description structurale et hiérarchique des contraintes stylistiques à l'œuvre dans un corpus : c'est un aspect de l'organisation des œuvres et des processus qui est bien réel, mais dont la pertinence n'est pas nécessairement perceptive.

Mais tout ceci n'enlève rien à la grandeur du livre de Lerdahl et Jackendoff. Nous sommes probablement en présence d'un des ouvrages de théorie et d'analyse musicale les plus remarquables du $\mathrm{XX}^{\mathrm{e}}$ siècle. Le modèle est composé de quatre éléments (pour un résumé, voir Lerdahl et Jackendoff 1983b) : la structure rythmique et la structure métrique qui conduisent à des groupements d'unités et qui servent de soubassement aux deux autres; la " time-span reduction ", qui montre, par le biais d'une structure arborescente, comment les relations harmoniques hiérarchisées sont dépendantes de la structure métricorythmique; la " prolongational reduction ", qui est la partie de l'ouvrage la plus directement inspirée de la symbologie schenkérienne. Si on excepte les relations de transformations entre motifs - un point sur lequel le modèle de Ruwet reste fondamental -, le modèle de Lerdahl et Jackendoff est probablement, du point de vue des aspects de la substance musicale traités, le plus complet qui ait été proposé pour l'analyse des œuvres tonales. On peut en juger en suivant tout au long du livre l'analyse récurrente du thème de la Symphonie $n^{\circ} 40$ de Mozart qui est abordé selon chacun des quatre points de vue. Au terme du parcours, les différents niveaux d'analyse peuvent être superposés afin de mettre en lumière leur interdépendance. Dans l'exemple 13, l'analyse métricorythmique, représentée par un système de points, et les découpages d'unités auxquels elle conduit sont donnés immédiatement en dessous de la portée. La réduction harmonique est détaillée dans les cinq systèmes $b$ à $g$ de la partie inférieure de l'exemple. L'arborescence qui y correspond est greffée sur la partition dans la partie supérieure. (La réduction prolongationnelle est donnée par les auteurs à la page suivante, avec l'arbre correspondant). 

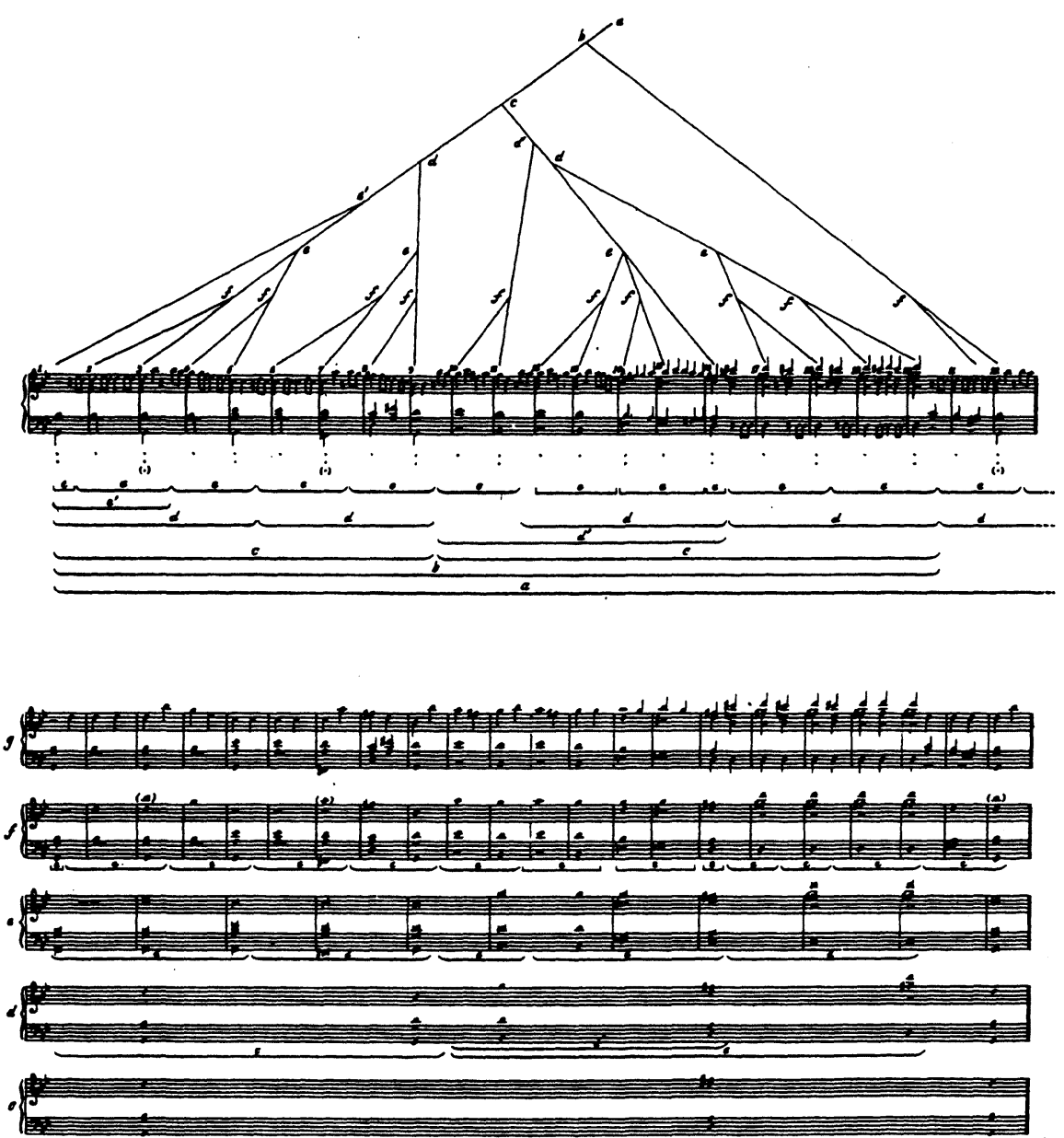

b

Exemple 13. Superposition d'analyses du thème de la Symphonie $n^{0} 40, \mathrm{~K} .550$ de Mozart (Lerdahl et Jackendoff 1983a, 259)

Le modèle prétend être " a formal description of the musical intuitions of a listener who is experienced in a musical idiom " (1983a, 1). Il n'est pas impossible qu'il s'agisse aussi de la compétence musicale d'un excellent élève de conservatoire ou de faculté de musique qui assigne à son auditeur idéal les mêmes capacités perceptives que les siennes. Dans ce cas, le modèle serait également pertinent du point de vue des prévisions perceptives incluses dans les stratégies poiétiques. J'ai consacré à ce problème difficile et délicat un petit article (Nattiez 1997a) auquel Lerdahl et Imberty ont répondu (Lerdahl 1997, Imberty 1997). Je ne peux ici que renvoyer à ce débat. En réalité, l'ouvrage de Lerdahl et Jackendoff fournit une excellente description des structures mor- 
phologiques d'une œuvre musicale à laquelle est attribuée une pertinence esthésique. En cela, il reste fondamentalement taxinomique ${ }^{13}$. Du reste, les 56 règles proposées n'ont pas toutes le même statut. Si certaines décrivent directement comment les structures d'une œuvre sont perçues, d'autres ont davantage pour fonction de guider l'analyse. La règle de proximité (GPR2) est un exemple du premier type : "Consider a sequence of four notes $n_{1} n_{2} n_{3} n_{4}$. All else being equal, the transition $n_{2}-n_{3}$ may be heard as a group boundary if the interval of time from the end of $n_{1}$ to the beginning of $n_{2}$ is greater than that from the end of $n_{1}$ to the beginning of $n_{2}$ and that from the end of $n_{3}$ to the beginning of $n_{4}$, etc. » (Lerdahl et Jackendoff 1983a, 45). Mais non seulement la règle nous indique comment un tel passage est perçu; nous sommes aussi en présence d'une règle de segmentation taxinomique, fondée non sur des critères paradigmatiques mais sur des critères syntagmatiques (l'environnement de $n_{2}-n_{3}$ ), propre à guider l'analyse qui se veut pertinente perceptivement. D'autres règles me semblent d'ordre plus directement méthodologique pour l'analyse et, de ce fait, s'apparentent davantage aux types de règles proposées par Ruwet : " Avoid analyses with very smaller groups - the smaller, the less preferable » (1983a, 43) (GPR 1) ou « Prefer a metrical structure in which beats of level $L_{i}$ that are stressed are strong beats of $L_{i} »(1983 \mathrm{a}, 79)$ (MPR 4), même si ces conseils adressés à l'analyste reposent, bien sûr, sur des règles censées décrire les stratégies perceptives. Mais dans un cas comme dans l'autre, ces règles peuvent être expérimentalement testées et amendées, ce qui est tout à fait conforme aux exigences normales de l'épistémologie. C'est d'ailleurs ce qui est arrivé, et pour le meilleur.

Car si l'ouvrage de Lerdahl et Jackendoff n'a pas connu une postérité riche du point de vue de l'analyse des œuvres stricto sensu, en revanche, la psychologie cognitive de la musique, en pleine effervescence comme on le sait, s'en est emparée. Irène Deliège (1987), en bonne psychologue expérientaliste de la musique, a vérifié la validité des règles de groupement perceptif auprès de sujets musiciens et non musiciens. On peut attendre encore davantage de la postérité de ce travail. Dans la mesure où 51 des 56 règles proposées sont données comme universelles (1983a, 345-52), Lerdahl et Jackendoff lancent aux ethnomusicologues un salutaire défi de taille qui n'a toujours pas été relevé. L'importance d'un travail ne se mesure pas seulement à son caractère novateur et à la valeur des modèles proposés, ce qui est le cas ici, mais aussi au champ d'investigations nouvelles qu'il propose.

\section{Le regole della musica}

Mario Baroni et Carlo Jacoboni avaient déjà proposé une grammaire générative de la partie de soprano des chorals de Bach, une des rares qui ait été réellement testée par ordinateur (1976), et ils ont travaillé à la génération de sa composante harmonique (Baroni et al. 1984). Mais c'est surtout leur ouvrage, Le regole della musica, cosigné avec Rossana Dalmonte, qui constitue, à ce jour, l'en-

$13 \mathrm{Ce}$ que j'appelle dans mon jargon l'esthésique inductive. 
treprise de grammaire stylistique la plus aboutie et la plus convaincante, prenant de surcroît comme corpus, non pas les énoncés relativement simples que fournit l'ethnomusicologie, mais une série de 31 airs d'un compositeur de l'époque baroque, Giovanni Legrenzi (Baroni, Dalmonte et Jacoboni 1999).

J'utilise le mot "stylistique " avec prudence ici, car, plus largement, il s'agit, pour les auteurs, de fournir " l'explication conceptuelle de la compétence grammaticale » (Baroni, Dalmonte et Jacoboni 1999, 13), c'est-à-dire les processus par lesquels le compositeur prend « les décisions quant aux rapports syntaxiques entre les notes et leur ordre de succession " et selon lesquels les auditeurs procèdent à " l'élaboration perceptive-cognitive de l'information sonore " $(1999,8$ n6). Est-ce vraiment le cas? Même si les auteurs rejettent le concept de " niveau neutre ", proposé par la théorie tripartite de Molino et Nattiez (1999, 8n6), je ne suis pas certain que l'entreprise démontre, pour chacune des 119 règles proposées, en quoi elles sont pertinentes du point de vue poïétique, esthésique ou des deux. Il faudrait pour cela appliquer aux descriptions morphologiques proposées une théorie du poïétique ou une théorie de l'esthésique qui leur assigne l'une ou l'autre de ces pertinences ou les deux (Nattiez 1997b, 17-18), de la même façon que, chez Lerdahl et Jackendoff, la théorie de la Gestalt est la théorie qui leur permet d'assigner une pertinence esthésique aux structures qu'ils dégagent. À mon sens, ces 119 règles résultent de magnifiques analyses... du niveau neutre! Je n'en dirai pas plus ici, sinon pour souligner que, pour ces raisons, $j$ 'ai quelques doutes quant à la légitimité du sous-titre du livre : " un panorama des mécanismes de communication ${ }^{14}$ ». Mais sans les règles proposées, il serait impossible de se prononcer sur ces pertinences, et c'est pourquoi nous sommes bien en présence d'un ouvrage décisif du point de vue de la compréhension du phénomène musical.

Je n'ai pas utilisé ici le terme "génératif ", même si l'entreprise repose sur le concept chomskyen de " compétence ", parce que l'objectif des auteurs n'est pas de produire des pastiches (Baroni, Dalmonte et Jacoboni 1999, 63), comme c'est souvent le cas des chercheurs cités précédemment (supra, n. 10), mais d'utiliser la production de faux comme moyen de vérifier les 119 règles proposées par la grammaire sur la base d'une analyse antérieure du corpus. En fait, ce qui nous est proposé, c'est le résultat de nombreux allers-retours " de l'analyse aux règles de l'analyse ", à la suite des générations d'énoncés musicaux qui, au cours de la recherche, ont montré aux auteurs ou l'insuffisance ou l'inadéquation des règles descriptives qu'ils avaient tiré de l'observation du corpus. En un mot, "l'analyse a comme objectif de trouver une régularité que la grammaire aura pour but de transformer en règles " (Baroni, Dalmonte et Jacoboni 1999, 51). En ce sens, ce travail est bien l'hériter des préoccupations des années 1960 et $1970:$ l'ensemble des règles repose sur un balayage systématique des récurrences caractéristiques du corpus qui font l'objet d'une description explicite testable par ordinateur. De plus, l'entreprise

14C'est l'auteur du présent article qui souligne. 
ne porte pas seulement sur un aspect limité du corpus (la monodie, comme c'est le cas le plus souvent avec les corpus de tradition orale), mais sur un ensemble de cinq dimensions essentielles de ces airs : la structuration du texte poétique (et son influence sur l'organisation musicale), la grande forme, l'organisation mélodique, l'harmonie et la ligne mélodique de la basse. Ce livre est, à ce jour, l'analyse d'inspiration linguistique la plus ample et la plus réussie qui porte sur un compositeur de musique tonale.

Qu'est-ce qui en fait l'importance et la réussite? D'abord, le fait que les règles proposées ne se confondent pas avec l'algorithme qu'il a fallu construire pour permettre à l'ordinateur de vérifier les règles. Du coup, malgré la minutie de détail, la lecture de l'ouvrage n'est pas entravée par la « technologie » d'une grammaire générative orthodoxe. Voici quelques exemples de ces règles : «Il n'est pas permis à deux tonalités relatives de se succéder ". Ou encore, «L'anacrouse ne comprend jamais plus de trois syllabes; le premier temps fort correspond à une des quatre premières syllabes avec accentuations de type 1 ou 2 ». Un dernier exemple : "Les cycles harmoniques complets sont formés par la succession des zones harmoniques fondamentales dominante-tonique, parfois précédées par la zone de la sous-dominante ou la zone de la tonique, ou par les deux dans n'importe quel ordre ".

On voit à travers ces quelques exemples que les règles proposées reposent d'abord sur l'identification de ce que Chomsky aurait appelé « les constituants immédiats » et que, de manière très opportune, les auteurs appellent les aspects " morphologiques ». Ici encore, comme chez Lerdahl et Jackendoff, tout le travail repose sur une taxinomie. En second lieu, la syntaxe de ces morphologies de base est appréhendée selon leur environnement (critères distributionnels) ou leur nombre (critères statistiques). C'est pourquoi, comme le plus souvent dans les pratiques les plus rigoureuses des sciences humaines, ces règles ont un caractère non pas déterministe mais probabiliste. Enfin, nous sommes bien en présence d'une approche scientifique, puisque ces règles sont suffisamment explicites pour que l'on puisse éventuellement démontrer qu'elles sont fausses, selon le critère de Popper. Fausses pour les auteurs qui les ont améliorées par essai et erreur tout au long de l'élaboration de la recherche. Fausses pour les musicologues qui s'emparent du livre et qui, confrontant les règles avec leurs propres observations du corpus des 31 airs, pourront être amenés à nuancer, corriger ou compléter les règles sur tel ou tel point. Parce qu'elles sont explicites, ces règles permettent d'enclencher un processus cumulatif de savoir.

Et de la même façon que les règles perceptives de Lerdahl et Jackendoff ont founi un extraordinaire matériau de base aux psychologues pour vérifier expérimentalement comment fonctionne la cognition musicale, les règles de Baroni, Dalmonte et Jacoboni pourraient bien apporter quelque chose de nouveau, non seulement à l'analyse musicale (à mon avis, cela est déjà acquis), mais à la musicologie historique. Car ce n'est pas le moindre mérite de ce livre que de proposer, dans la troisième partie, après avoir constaté que la partie la plus neuve, du point de vue de la connaissance musicale, touche à l'organisa- 
tion de la mélodie, un vaste balayage de l'histoire de la mélodie occidentale, du grégorien à Hindemith. Certains traits généraux des différentes périodes sont confrontés aux règles régissant la phrase mélodique chez Legrenzi. Non seulement voit-on se dessiner une perspective diachronique pour l'analyse musicale fondée sur des critères historiques, mais on peut deviner que, si cette tendance se développait, la manière d'écrire l'histoire de la musique s'en trouverait transformée.

\section{CONCLUSIONS}

La prolifération d'analyses ponctuelles, inspirées des modèles de la linguistique structurale, de Chenoweth à Becker, de Stefani à Nattiez, suffiraient pour affirmer que, à partir des années 1960 , et pour une longue période, une tendance importante s'est glissée dans le champ de l'analyse musicale. L'existence de quelques grands livres, ceux de Ruwet, Arom, Lerdahl et Jackendoff, et Baroni, Dalmonte et Jacoboni, nous convainquent que nous sommes désormais en présence d'un courant nouveau dans l'histoire de la musicologie.

Ce n'est pas une raison pour afficher un triomphalisme de mauvais aloi. D'abord, parce que des approches hiérarchiques, rigoureuses et novatrices, il en a existé d'autres au $\mathrm{XX}^{\mathrm{e}}$ siècle, qui ne sont pas nées de la rencontre avec la linguistique. Qu'il suffise de citer les noms de Schenker, Schoenberg, Réti, Forte, C. Deliège, Meyer ou Narmour, sans parler des travaux d'analyse faisant appel à l'ordinateur. La différence, capitale au demeurant, réside dans la rédaction de règles explicites, ou l'utilisation de ces règles, qui sont le trait commun à tous les grands ouvrages que j'ai cités : règles pour le contrôle raisonné de l'analyse, ou règles pour présenter de façon rigoureuse les résultats de l'analyse. Au-delà du lien qui a longtemps relié sémiologie musicale et linguistique, il n'y a aucune raison, comme je l'ai signalé plus haut, de ne pas combiner des modèles d'analyse d'inspiration linguistique avec ceux qui viennent d'ailleurs. Les modèles d'inspiration linguistique seront ainsi complétés par le traitement d'aspects de la musique qui leur avaient trop longtemps échappés (par exemple, la prolongation). À l'inverse, la rigueur introduite par les modèles linguistiques pourront amener à reformuler les autres modèles. C'est ce qui s'est produit lorsque Lerdahl et Jackendoff se sont inspirés de Schenker. Il y a donc encore beaucoup à faire. Et notamment du point de vue du lien entre les structures musicales, le monde, les stratégies compositionnelles et perceptives, l'histoire et la culture.

Au travers du parcours que je viens de proposer, j'ai essayé de montrer que les analyses musicales immanentes avaient très souvent débordé sur d'autres dimensions : sur les significations affectives, imageantes ou dénotatives qui leur sont reliées, sur les stratégies compositionnelles, sur les stratégies perceptives. En ce qui concerne le lien entre l'analyse des structures musicales et les deux autres pôles (poïétique et esthésique) de la tripartition sémiologique, quelques articles ponctuels ont montré à quels résultats on pouvait parvenir dans cette direction (Nattiez 1992a, 1997b, 1998). Mais c'est la thèse, à paraître, de Luiz Paulo Sampaio (1999) qui en fait la démonstration exemplaire 
et complète à propos d'un corpus complexe, les Variationen, op. 27, de Webern.

Il semble qu'avec les recherches récentes d'étudiants montréalais, une nouvelle époque de l'utilisation des modèles linguistiques dans l'analyse musicale soit en train de naître. Elles ont en commun de combiner entre eux l'utilisation des différents modèles et de les intégrer dans une perspective musicologique plus vaste, et pas seulement pour faire la démonstration de l'efficacité d'un modèle particulier. Ainsi Guy Marchand (2003), pour établir les liens entre la petite fugue d'une suite pour luth (BWV 997) et le chœur d'entrée de la Passion selon Saint Matthieu, s'est-il astreint à l'analyse paradigmatique des 200 cantates d'église de Bach, ce qui lui permet de montrer que le cantor de Leipzig avait recours au nombre d'or bien plus qu'on ne le croyait; mais c'est pour établir des liens entre ces résultats et une investigation beaucoup plus large qui met en branle la rhétorique, le symbolisme et l'herméneutique luthérienne. Dans ce qui apparaît désormais comme le premier manuel d'analyse des musiques électroacoustiques, Stéphane Roy (2003) fait non seulement le bilan critique des analyses d'inspiration phonologique de ce type de musique, mais propose l'élaboration de modèles originaux inspirés de l'analyse paradigmatique, de la perspective générative de Lerdhal-Jackendoff et du modèle implicatif de Meyer, tout en proposant son propre modèle $d$ 'analyse fonctionnelle. Et je m'en voudrais de ne pas signaler deux travaux d'envergure qui ne manqueront pas de faire l'objet de publications. Frédéric Léotar (2004) a pu proposer, à partir de l'analyse paradigmatique d'une centaine de chants huchés, une grammaire générative à pertinence poïétique qui montre quels mécanismes cognitifs les Touvas et les Ouzsbèkes mettent en branle pour produire ces chants. Enfin, Sylveline Bourion prépare actuellement un travail majeur sur les duplications debussystes : confrontant les propositions initiales de Ruwet, en 1962, à une analyse approfondie de toutes les mélodies pour voix et piano de Debussy, elle est amenée à redéfinir le fonctionnement et la fonction du phénomène de duplication chez ce compositeur, en même temps qu'elle en élabore une stylistique particulièrement novatrice. Pour cela, elle passe, elle aussi, de l'analyse paradigmatique des œuvres particulières à la construction d'une grammaire générative de la duplication chez Debussy ${ }^{15}$.

Si nous nous plaçons maintenant du point de vue des relations entre analyse et histoire, le bouillonnement sémio-linguistique des années $1960 \mathrm{a}$ pu faire l'objet de critiques du côté des historiens de la musique (Schneider 1980; Powers 1980). L'ouvrage consacré aux airs de Legrenzi ouvre une voie royale à la connexion entre l'analyse musicale rigoureuse et l'histoire. Si l'on prend quelques grands classiques de l'histoire de la musique, comme les ouvrages de Bukofzer ou d'Einstein, on est frappé par la rareté ou l'absence d'exemples musicaux. C'est tout simplement, comme me le suggère Molino, parce que, en 1947, la conception de l'œuvre musicale comme organisation structurale hiérarchisée n'était pas encore à l'ordre du jour. Dans le cas du Classical Style 
de Rosen (1971) où, au contraire, il y a de un à trois exemples musicaux par page, l'auteur va piocher dans l'immensité de ce corpus ce qui lui semble convenir à sa thèse ("Le style classique, c'est le drame »), sans passer par une description systématique de chacune des œuvres et des styles. Il n'en va pas autrement dans les travaux du " music criticism " anglo-saxon où l'analyse musicale est très présente, tout en refusant les modèles trop rigoureux de la " music theory ", comme y insiste Kerman, son porte-parole autoproclamé (Kerman 1985, chap. 3). À partir du moment où l'on va disposer d'analyses locales, mais de plus en plus nombreuses et de plus en plus vastes, de styles particuliers, il ne sera plus possible, comme le fait au fond l'histoire traditionnelle de la musique, de s'en tenir à expliquer par de larges traits externes (biographiques, événementiels, sociaux, culturels) l'émergence, l'existence ou la transformation de tel ou tel genre. Il sera nécessaire de pénétrer dans le détail des styles et poser en des termes nouveaux les rapports entre les différents niveaux hiérarchiques et paramétriques des structures musicales, et l'histoire de la musique.

Du côté de l'ethnomusicologie, l'irruption des modèles linguistiques dans les années 1960 avait été fortement critiquée, parce qu'elle laissait de côté le lien entre les productions musicales et l'environnement culturel (Feld 1974; voir aussi la réplique de Herndon [1974]). Il y a fort à parier qu'au-delà de la vision purement ethnomusicologique d'un Arom ou de la vision purement ethnomusicologique de la grande majorité des approches contemporaines de cette discipline, il y aura place, désormais, pour la construction d'une véritable ethno-musicologie, c'est-à-dire à une approche qui réussira à déterminer à quel niveau de la structure musicale se situent effectivement les liens entre les constituants des pièces musicales et la culture (pour une présentation de ma position actuelle à ce sujet, voir Schulte-Tenckhoff 1999).

Il est donc probable qu'on assistera à un élargissement de plus en plus grand de l'horizon par rapport auquel les structures musicales seront analysées. On peut déjà parier que, au-delà des sciences neuro-psychologiques, la biologie de la musique viendra s'ajouter aux disciplines qui prendront en charge la globalité du fait musical; et il n'est pas impossible que, de cette rencontre, jaillisse un bouleversement complet de l'analyse musicale. Mais si le développement des approches musicales rigoureuses se poursuit, même dans des cadres qui déborderont l'immanence initiale de l'approche, il ne faudra jamais oublier la grande leçon que, d'ores et déjà, la rencontre des modèles linguistiques et de l'analyse musicale nous apporte : ne jamais quitter le texte ${ }^{16}$ !

16 Cet article est une version profondément révisée et très augmentée de deux contributions antérieures (Nattiez 1988, 1992b). Il a été rédigé à la demande du professeur Enrico Fubini qui l'a fait paraitre en italien et en anglais dans la Rivista italiana di musicologia 35 , nos $1-2(2001)$ : $321-410$, dans un numéro consacré aux tendances de la musicologie à la fin du $\mathrm{XX}^{\mathrm{e}}$ siècle. L'original français a été révisé et mis à jour et je remercie Serge Lacasse et la Revue de musique des universités canadiennes de me donner la possibilité de le faire paraître. 


\section{RÉFÉRENCES}

Arom, Simha. 1969. "Essai d'une notation des monodies à des fins d'analyse ». Revue de musicologie 55, $\mathrm{n}^{0} 2: 172-216$.

- 1970. Conte et chantefables Ngbaka Ma'bo. Paris : SELAF.

1985. Polyphonies et polyrythmies instrumentales d'Afrique centrale: structure et méthodologie. 2 vol. Paris : SELAF.

Asch, Michael. 1975. "Social Context and the Musical Analysis of Slavey Drum Dance Songs ». Ethnomusicology 19, $\mathrm{n}^{0} 2: 245-57$.

Austerlitz, Robert. 1983. "Meaning in Music : Is Music like Language and if so How? ». American Journal of Semiotics 2, $\mathrm{n}^{0} 3: 1-12$.

Ayrey, Craig. 1985. " Deconstructing Debussy ». Communication, congrès annuel, août. Melbourne : Australian Musicological Society.

Baroni, Mario, Rosella Brunetti, Laura Callegari et Carlo Jacoboni. 1984. « A Grammar for Melody: Relationships between Melody and Harmony ". Dans Baroni et Callegari 1984, 201-18.

Baroni, Mario, et Laura Callegari, dir. 1984. Musical Grammars and Computer Analysis. Quaderni della rivista italiana di musicologia 8. Florence : Olschki editore. Atti del convegno, Modène, 4-6 octobre 1982.

Baroni, Mario, Rossana Dalmonte et Carlo Jacoboni. 1999. Le regole della musica, Indagine sui meccanismi della comunicazione. Turin : Edizioni di Torino. Trad. angl. A Computer-Aided Inquiry on Music Communication. The Rules of Music. "Studies in the History and Interpretation of Music ", vol. 92. Lewingston, NY : Edwin Mellen Press, 2003. Préface de JeanJacques Nattiez. Trad. fr. en préparation.

Baroni, Mario, et Carlo Jacoboni. 1976. Verso una Grammatica della Melodia. Bologne : Antiquae Musicae Italicae Studiosi, Università Studi di Bologna. Trad. angl. Proposal for a Grammar of Melody. Montréal : Presses de l'Université de Montréal, 1978.

Beaudry, Nicole. 1983. "Le langage des tambours dans la cérémonie vaudou haïtienne ", Revue de musique des universités canadiennes, $\mathrm{n}^{0} 4: 125-40$.

Becker, Judith, et Alton Becker. 1979. « A Grammar of the Musical Genre Srepegan ", Journal of Music Theory 23, $\mathrm{n}^{0} 1: 1-43$.

Becking, Gustav. 1933. "Der musikalische Bau des montenegrinischen Volksepos ", Archives néerlandaises de phonétique expérimentale 7-9 : 144-53. Proceedings of the First International Congress of Phonetic Science, Amsterdam, 3-8 juillet 1932.

Bent, Ian. 1980. « Analysis ». Dans Sadie 1980, I:340-88. Rééd. avec Willam Drabkin, Norton/Grove, Handbooks in Music, New York: W.W. Norton, 1987. Trad. fr. de la version de 1987, L'Analyse musicale. Histoire et méthodes. Nice : Main-d'œuvre, 1998. Rééd. avec Anthony Pople, dans Sadie 2001, I:526-89.

Bernstein, Leonard. 1982. La question sans réponse. Paris : Laffont. Orig. angl. The Unanswered Question. Cambridge : Harvard University Press, 1976. 
Bierwisch, Manfred. 1979. Musik und Sprache : Überlegungen zu ihrer Struktur und Funktionsweise. Leipzig : Peters.

Blacking, John. 1971a. " Deep and Surface Structures in Venda Music ". Yearbook of the International Folk Music Council 3 : 69-98.

- 1971b. "Towards a Theory of Musical Competence ». Dans Man : Anthropological Essays Presented to O.A. Raum, sous la dir. de De Jager, 19-34. Cape Town : C. Struik.

- 1973. How Musical is Man? Seattle : University of Chicago Press. Trad. fr. Le sens musical. Paris : Éditions de Minuit, 1980.

Boilès, Charles. 1967. «Tepehua Thought-Song : a Case of Semantic Signaling ». Ethnomusicology 11, no $3: 267-92$. Trad. fr. "Les chants instrumentaux des Tepehuas : un exemple de transmission musicale de significations ». Musique en jeu, $\mathrm{n}^{0} 12$ (1973) : 81-89.

- 1973a. "Reconstruction of Proto-Melody ». Yearbook for InterAmerican Musical Research $9:$ 45-63.

$34-41$.

- 1975. "La signification dans la musique de film ». Musique en jeu, $\mathrm{n}^{0} 19$ : 69-85. Orig. angl. Versus, $\mathrm{n}^{0} 13$ (1976) : 49-61.

. 1982. «A Paradigmatic Test of Acculturation ». Dans Cross-Cultural Perspectives on Music, sous la dir. de Robert Falck et Timothy Rice, 53-78. Toronto : University of Toronto Press.

Boudon, Raymond. 1968. À quoi sert la notion de structure? Paris : Gallimard. Boulez, Pierre. [1961] 1995. "L'esthétique et les fétiches ". Dans Points de repère I: Imaginer, sous la dir. de Jean-Jacques Nattiez et Sophie Galaise, 491-505. Paris : Christian Bourgois éditeur. Première publ. dans Panorama de l'art musical contemporain, sous la dir. de Claude Samuel, 401-15. Paris : Gallimard, 1962.

Bourion, Sylveline. 2004. "Pour une grammaire générative de la duplication dans les derniers cycles de mélodies pour voix et piano de Debussy $"$. Musurgia : analyse et pratique musicales (à paraître).

Brandily, Monique. 1976. "Un chant du Tibesti (Tchad) ». Journal des africanistes $46, \mathrm{n}^{\text {os }} 1-2: 127-92$.

Brediceanu, Mihai. 1975. "Sur les transformations topologiques et les mécanismes génératifs en musique $»$. Semiotica $15, \mathrm{n}^{\circ} 1: 58-70$.

Bright, William. 1963. "Language and Music : Areas for Cooperation ». Ethnomusicology 7, $\mathrm{n}^{0} 1: 26-32$. Trad. fr. " Points de contact entre langage et musique ». Musique en jeu, $\mathrm{n}^{0} 5$ (1971) : 67-74.

Bukofzer, Manfred. 1947. Music in the Baroque Era. New York : W. W. Norton.

Camilleri, Lelio. 1984. " A Grammar of the Melodies of Schubert's Lieder ». Dans Baroni et Callegari 1984, 229-36.

Cazimir, Bogdan. 1975. "Sémiologie musicale et linguistique mathématique ». Semiotica $15, \mathrm{n}^{0} 1: 48-57$. 
Chandola, Anoop. 1970. "Some System of Musical Scales and Linguistic Principles ». Semiotica $2, \mathrm{n}^{\circ} 2: 135-50$.

- 1977. Folk Drumming in the Himalayas. A linguistic Approach to Music. New York : American Musicological Society Press.

Chenoweth, Vida. 1966. "Song Structure of a New Guinea Highlands Tribe». Ethnomusicology 12, no 3 : 285-97.

- 1972. Melodic Perception and Analysis. Papouasie-Nouvelle-Guinée : Summer Institute of Linguistics.

- 1979. The Usarufas and their Music. Dallas : Summer Institute of Linguistics, Museum of Anthropology.

Chenoweth, Vida, et Darlene Bee. 1971. "Comparative-Generative Models of a New Guinea Melodic Structure ». American Anthropologist 73, $\mathrm{n}^{\circ} 3$ : 773-82.

Chiarucci, Henri. 1973. «Essai d'analyse structurale d'œuvres musicales ». Musique en jeu, $\mathrm{n}^{\circ} 12: 11-43$.

Chomsky, Noam. 1957. Syntactic Structures. La Haye : Mouton. Trad. fr. Structures syntaxiques. Paris : Éditions du Seuil, 1969.

-1965. Aspects of the Theory of Syntax. Cambridge : MIT Press. Trad. fr. Aspects de la théorie de la syntaxe. Paris : Éditions du Seuil, 1971.

Chomsky, Noam, et George A. Miller. 1963. "Introduction to the Formal Analysis of Natural Languages ». Dans Handbook of Mathematical Psychology, sous la dir. de R. Duncan Luce, Robert R. Bush et Eugene Galanter, chap. 2. New York : John Wiley.

Cogan, Robert. 1984. New Images of Musical Sound. Cambridge : Harvard University Press.

Collectif. 1980. Computer Music Journal 9, $\mathrm{n}^{\circ} 2$.

Collectif. 1991. " Analyse et expérimentation. En hommage à Simha Arom et à son équipe ". Analyse musicale, $\mathrm{n}^{\circ} 23$ (avril) : 21-49.

Cooper, Robin. 1973. "Propositions pour un modèle transformationnel de description musicale ». Musique en jeu, $\mathrm{n}^{0} 10: 70-88$.

Dehoux, Vincent. 1986. Chants à penser gbaya (Centrafrique). Paris : SELAF. Deliège, Célestin. 1965. "La musicologie devant le structuralisme ». L'Arc, $\mathrm{n}^{\circ} 26: 45-52$.

. 1975a. "Webern : op. $10, \mathrm{n}^{\circ} 4$, un thème d'analyse et de réflexion ». Revue de musicologie 20, $\mathrm{n}^{\text {os }} 1-4: 21-42$.

- 1975b. "Théorie et pratique de l'analyse musicale ». Dans Stefani $1975,151-71$.

- 1984. Les fondements de la musique tonale. Paris : Lattès.

Deliège, Irène. 1987. "Grouping Conditions in Listening to Music : An Approach to Lerdahl and Jackendoff's Grouping Preference Rules ». Music Perception 4, $\mathrm{n}^{\circ} 4$ : 325-60.

Desroches, Monique. 1980. "Validation empirique de la méthode sémiologique en musique : le cas des indicatifs de tambour dans les cérémonies indiennes en Martinique ". Yearbook of the International Folk Music Council $12: 67-76$. 
1982. "Semiotic Analysis and the Music of Tamil Religious Ceremonies in Martinique ». Dans Three Musical Analyses, Jean-Jacques Nattiez, Marcelle Guertin et Monique Desroches, Monographs, Working Papers and Prepublications (Toronto Semiotic Circle) 4, 59-70. Toronto : Victoria University, 1982.

1996. Tambours des dieux : musique et sacrifice d'origine tamoule en Martinique. Montréal : L'Harmattan.

Dosse, François. 1991-92. Histoire du structuralisme. Vol. I, Le champ du signe, 1945-1966. Vol. II, Le chant du cygne, 1967 à nos jours. Paris : Éditions la découverte.

Dufrenne, Mikel. 1953. Phénoménologie de l'expérience esthétique. Paris : Presses universitaires de France.

- 1966. "L'art est-il langage? ». Revue d'esthétique 19, $\mathrm{n}^{\circ} 1$ (janv.mars) : 1-42. Repris dans Esthétique et philosophie, 74-122. Paris : Klincksieck, 1967.

- 1968. "L'art est-il langage? (Syntaxe et sémantique dans l'art) ». Rivista di estetica $13, \mathrm{n}^{0} 2: 171-77$.

Dunsby, Jonathan. 1982. «A Hitch Hicker's Guide to Semiotic Music Analysis ». Music Analysis $1, \mathrm{n}^{0} 3: 235-42$.

Dunsby, Jonathan, et Arnold Whittall. 1988. Music Analysis in Theory and Practice. Londres : Faber.

Einstein, Alfred. 1947. Music in the Romantic Era. New York: W. W. Norton. Ellis, Alexander J. 1885. "On the Musical Scales of Various Nations ». Journal of the Society of the Arts $33: 485-527$.

Everist, Mark. 1987. "Introduction to Nicolas Ruwet, "Methods of Analysis in Musicology" ". Trad. angl. de Ruwet 1966. Music Analysis 6, nos 1-2 : 3-9.

Feld, Steven. 1974. "Linguistics and Ethnomusicology ». Ethnomusicology $18, \mathrm{n}^{0} 2: 197-217$.

Forte, Allen. 1959. "Schenker's Conception of Musical Structure ». Journal of Music Theory 3, $\mathrm{n}^{0} 1: 1-30$.

Fubini, Enrico. 1973. Musica e linguaggio nell'estetica contemporanea. Turin : Einaudi.

Giomi, Francesco, et Marco Ligabue. 1988. Software di generazione e studio di musica jazz. Florence : CNUCE/CNR (Rapporto interno C88-06).

- 1998. « Evangelisti's Composition Incontri di Fasce Sonore at WDR : Aesthesic-Cognitive Analysis in Theory and Practice ». Journal of New Music Research 27, $\mathrm{n}^{\text {os }}$ 1-2 : 120-45, Analysis of Electroacoustic Music, sous la dir. de Lelio Camilleri et Denis Smalley.

Goldman, Jonathan. 1998. "Analyzing Pierre Boulez. Notes on Anthèmes : "Creating a Labyrinth Out of Another Labyrinth" ". Mémoire de maitrise, Université de Montréal. En ligne, Understanding Pierre Boulez's Anthèmes. "Creating a Labyrinth Out of Another Labyrinth". http://www.andante.com/reference/academy/thesis/anthemsth esis.pdf. 
Goldman, Richard Franko. 1965. Harmony in Western Music. New York : W. W. Norton.

Gross, Maurice. 1968. Grammaire transformationnelle du français : syntaxe $d u$ verbe. Paris : Larousse.

1977. Grammaire transformationnelle du français : syntaxe du nom. Paris : Larousse.

- 1979. «On the Failure of Generative Grammar ». Language 55, no 4 (déc.) : 859-85.

Guertin, Marcelle. 1974. Sémiologie et interprétation : quelques aspects $d$ '̂le de feu 2 de Messiaen. Université de Montréal, Groupe de recherches en sémiologie musicale 5. Montréal : Faculté de musique de l'Université de Montréal.

- 1981. "Différence et similitude dans les Préludes pour piano de Debussy ". Revue de musique des universités canadiennes, $\mathrm{n}^{0} 2: 56-83$.

- 1990. De la lecture à l'audition d'un texte musical : une étude des thèmes dans le Livre I des Préludes pour piano de Debussy. Préface de Jean-Jacques Nattiez. Montréal : Les Presses de l'Université de Montréal. Hagège, Claude. 1982. La structure des langues. Paris : Presses universitaires de France.

Hanslick, Eduard. 1854. Vom Musikalisch-Schönen. Leipzig : Rudoph Weigel. Trad. fr. Du beau dans la musique. Préface de Jean-Jacques Nattiez. Paris : Christian Bourgois éditeur, 1986.

Harris, Zellig. 1951. Structural Linguistics. Chicago : The University of Chicago Press.

Harweg, Roland. 1967. «Sprache und Musik : immanent und zeichentheoretisch gesehen ». Poetica $1: 390-414$. Trad. angl. " Language and Music : An Immanent and Sign Theoretic Approach ». Foundations of Language 4 (1968) : 270-81. Trad. fr. « Langage et musique : une approche immanente et sémiotique ». Musique en jeu, $\mathrm{n}^{\circ} 5$ (1971) : 19-30.

Herndon, Marcia. 1974. " Analysis : Herding of Sacred Cows? ». Ethnomusicology $18, \mathrm{n}^{0} 2: 219-62$.

-1975. «Le modèle transformationnel en linguistique : ses implications pour l'étude de la musique ». Semiotica 15, no $1: 71-82$.

Hornbostel, Erich Moritz. 1975. Hornbostel Opera Omnia. Vol. 1. La Haye : Nijhoff.

Hughes, David W. 1991. "Grammars of Non Western Musics : a Selective Survey ». Dans Representing Musical Structure, sous la dir. de Peter Howell, Robert West et Ian Cross, 327-62. Londres : Academic Press.

Imberty, Michel. 1997. « Epistemic Subject, Historical Subject, Psychological Subject : Regarding Lerdahl and Jackendoff's Generative Theory of Tonal Music. A Response to Jean-Jacques Nattiez ». Dans Perception and Cognition of Music, sous la dir. de Irène Deliège et John A. Sloboda, 429-32. Hove : Psychology Press.

Jakobson, Roman. 1932. "Musikwissenschaft und Linguistik ". Prager Presse 7 (déc.). Repris dans Selected Writings, 551-53. La Haye : Mouton, 
1971. Trad. fr. "Musicologie et linguistique ». Musique en jeu, $\mathrm{n}^{0} 5$ : 57-60.

. 1970. "Language in Relation to Other Communication Systems ». Dans Linguaggi nella società e nella tecnica, 3-16. Milan : Edizioni di Comunità.

1973. «Le langage en relation avec les autres systèmes de communication ». Dans Essais de linguistique générale II. Rapports internes et externes du langage, 91-103. Paris : Minuit, 1973. Trad. fr. de Jakobson 1970.

Johnson-Laird, Philip N. 1991. « Jazz Improvisation : A Theory of Computational Level ». Dans Representing Musical Structure, sous la dir. de Peter Howell, Robert West et Ian Cross, 291-326. Londres : Academic Press.

Kassler, Michael. 1975. Proving Musical Theorems I : The Middleground of Heinrich Schenker's Theory of Tonality. Technical Report no 103. Department of Computer Science, The University of Sydney.

- 1977. "Explication of the Middleground of Schenker Theory of Tonality $»$. Miscellanea Musicologica $9: 72-81$.

Keiler, Allan. 1977. « The Syntax of Prolongation: Part 1 ». Theory Only 3, n 5 : 3-27.

1978a. "Bernstein's The Unanswered Question and the Problem of Musical Competence ». The Musical Quarterly 64, $\mathrm{n}^{0} 2: 195-222$.

- 1978b. « The Empiricist Illusion : Narmour's beyond Schenkerism », Perspectives of New Music 17, $\mathrm{n}^{0} 1: 161-95$.

- 1981. "Two Views of Musical Semiotics ». Dans Steiner 1981, 138-68.

Kerman, Joseph. 1985. Musicology. Londres : Fontana Press.

Kippen, James. 1987. " An Ethnomusicological Approach to the Analysis of Musical Cognition ». Music Perception 5, $\mathrm{n}^{\circ} 2$ : 173-96.

Kippen, James, et Bernard Bel. 1992. " Modeling Music with Grammars. Formal Language Representation in the Bol Processor ». Dans Computer Representations and Models in Music, sous la dir. d'Alan Marsden et Anthony Pople, 207-38. Londres : Academic Press.

Kundera, Milan. 1984. "The Tragedy of Central Europe ». The New York Review of Books 31, $\mathrm{n}^{0} 17$ (26 avril) : 33-38.

Labussière, Annie. 1992. "Die alte Weise". Une analyse sémiologique du solo de cor anglais du $3^{\mathrm{e}}$ acte de Tristan et Isolde ». Analyse musicale, $\mathrm{n}^{\circ} 27: 30-53$.

Laske, Otto E. 1972-73. Introduction to a Generative Theory of Music. Sonological Reports, $\mathrm{n}^{0}$ 1B. Utrecht : Utrecht State University, Institute of Sonology.

Lees, Robert B. 1960. The Grammar of English Nominalizations. La Haye : Mouton.

Lenneberg, Erich Heinz. 1967. Biological Foundations of Language. New York : John Wiley. 
Leotar, Frédéric. 2004. « Les mélodies huchées touvas et ouzbèkes : aspects compositionnels et dimension culturelle ». Thèse de doctorat, Université de Montréal.

Lerdahl, Fred. 1997. " Composing and Listening : A Reply to Nattiez ». Dans Perception and Cognition of Music, sous la dir. d'Irène Deliège et John A. Sloboda, 421-28. Hove : Psychology Press.

Lerdahl, Fred, et Ray Jackendoff. 1983a. A Generative Theory of Tonal Music. Cambridge, Mass. : M.I.T. Press.

- 1983b. "An Overview of Hierarchical Structure in Music ». Music Perception 1, $\mathrm{n}^{0} 2$ : 229-47.

Lévi-Strauss, Claude. 1964. Le cru et le cuit. Paris : Plon.

Levy, Morten. 1975. "On the Problem of Defining Musical Units ». Dans Stefani 1975, 135-49. Trad. fr. «Sur le problème de la définition des unités musicales ». Semiotica 15, $\mathrm{n}^{0} 1$ (1975) : 58-70.

Lidov, David. 1975. On Musical Phrase. Université de Montréal, Groupe de recherches en sémiologie musicale 1. Montréal : Faculté de musique de l'Université de Montréal.

- 1978. "Nattiez's Semiotics of Music ". Canadian Journal of Research in Semiotics $5, \mathrm{n}^{0} 2: 13-54$.

Lidov, David, et James Gabura. 1973. «A Melody Writing Algorithm Using a Formal Language Model ". Computer Studies in the Humanities 4, $n^{\text {os }} 3-4: 138-48$.

Lindblom, Björn, et Johann Sundberg. 1970. « Towards a Generative Theory of Melody ». Swedish Journal of Musicology 52 : 70-88.

- 1972. "Music Composed by a Computer Program ». Speech Transmission Laboratory : Quarterly Progress and Status Report 4 : 20-28.

Lortat-Jacob, Bernard. 1975. "Quelques problèmes généraux d'analyse musicale ». Revue de musicologie 61, $\mathrm{n}^{\circ} 1: 3-34$.

- dir. 1987. L'improvisation dans les musiques de tradition orale. Paris : SELAF.

Mâche, François-Bernard. 1971. "Méthodes linguistiques en musicologie ». Musique en jeu, $\mathrm{n}^{0} 5: 75-91$.

. 1983. Musique, mythe, nature ou les dauphins d'Arion. Paris : Klincksieck.

1986. "Les procédures d'analyse sémiologique ". International Review of the Aesthetics and Sociology of Music 17, $\mathrm{n}^{\circ} 2: 203-14$.

MacKay, John. 1988. " Aspects of Post-Serial Structuralism in Berio's Sequenza $I V$ and $V I »$. Interface $17, \mathrm{n}^{\circ} 4: 223-40$.

Marchand, Guy. 2003. Bach ou la Passion selon Jean-Sébastien : de Luther au nombre d'or. Préface de Jean-Jacques Nattiez. Paris : L'Harmattan.

Menezes, Flo. 1993. Luciano Berio et la phonologie : une approche jakobsonienne de son cuvre. Francfort : Peter Lang.

Merriam, Alan P. 1964. The Anthropology of Music. Evanston : Northwestern University Press. 
Meyer, Leonard B. 1967. Music, the Arts, and Ideas. Chicago : University of Chicago Press.

- 1973. Explaining Music. Berkeley : University of California Press.

Middleton, Richard. 1990. Studying Popular Music. Philadelphie : Open University Press.

Molino, Jean. 1975. "Fait musical et sémiologie de la musique ». Musique en jeu, $\mathrm{n}^{0} 17: 37-62$.

- 1989. "Musique et machine ». Dans Actes du colloque "Structures musicales et assistance informatique ", 141-54. Marseille : Laboratoire "Musique et informatique ".

Monelle, Raymond. 1992. Linguistics and Semiotics in Music. Chur : Harwood Academic Publishers.

Morin, Élisabeth. 1979a. Les structures mélodiques et rythmiques d'une fugue

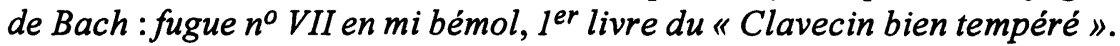
Préface de Jean Molino. Université de Montréal, Groupe de recherches en sémiologie musicale 7. Montréal : Faculté de musique de l'Université de Montréal.

- 1979b. Essai de stylistique comparée : les variations de William Byrd et John Tomkins sur " John Come Kiss me Now ». 2 vol. Montréal : Presses de l'Université de Montréal.

Mounin, Georges. 1968. Clefs pour la linguistique. Paris : Seghers.

Nattiez, Jean-Jacques, dir. 1971. "Sémiologie de la musique », Musique en jeu, $\mathrm{n}^{0} 5$.

- 1972. " La linguistique, voie nouvelle pour l'analyse musicale?». Cahiers canadiens de musique, $\mathrm{n}^{0} 4: 101-15$.

— dir. 1973a. "Analyse, méthodologie, sémiologie ». Musique en jeu, $\mathrm{n}^{0} 10$.

— dir. 1973b. " Autour de Lévi-Strauss ». Musique en jeu, $\mathrm{n}^{0} 12$.

— dir. 1973c. «Per una semiotica della musica ». Versus $5, \mathrm{n}^{\circ} 1$.

- 1973d. De l'analyse taxinomique à la caractérisation stylistique (Claude Debussy: Syrinx). Université de Montréal, Groupe de recherches en sémiologie musicale 2. Montréal : Faculté de musique de l'Université de Montréal. Trad. angl. [version révisée] « From Taxomic Analysis to Stylistic Characterization ". Actes du premier congrès international de sémiotique musicale, 83-110. Pesaro : Centro di Iniziativa Culturale, 1975. Trad. it. [version révisée] « Syrinx di Debussy : un'analisi paradigmatica ». Dans Il senso in musica. Antologia di semiotica musicale, sous la dir. de Luca Marconi et Gino Stefani, 93-137. Bologne : CLUEB.

-1973e. "Trois modèles pour l'analyse musicale ». Musique en jeu, $\mathrm{n}^{0} 10: 3-11$.

- 1975a. Fondements d'une sémiologie de la musique. Paris : Union Générale d'Éditions.

— dir. 1975b. « De la sémiologie à la sémantique musicales 》. Musique en jeu, $\mathrm{n}^{\circ} 17$. 
dir. 1975 c. "Unités et transformations musicales ». Semiotica 15, $\mathrm{n}^{0} 1$.

1975d. Densité 21,5 de Varèse : essai d'analyse sémiologique. Université de Montréal, Monographie / Groupe de recherches en sémiologie musicale 2. Montréal : Faculté de musique de l'Université de Montréal. Trad. angl. [version révisée] "Varese's Density 21.5: A study in Semiological Analysis ». Music Analysis 1, $\mathrm{n}^{0} 3$ (oct. 1982) : 243-340.

1983. "Some Aspects of the Study of Inuit Vocal Games ». Ethnomusicology 27, $\mathrm{n}^{0} 3: 457-75$. Version fr. révisée "Sémiologie des jeux vocaux Inuit ». Semiotica $66, \mathrm{n}^{\text {os }} 1-3$ (1987) : 259-78.

-1987. Musicologie générale et sémiologie. Paris : Christian Bourgois éditeur. Trad. angl. [version révisée] Music and Discourse: Toward a Semiology of Music. Princeton : Princeton University Press, 1990.

1988. "Réflexions sur le développement de la sémiologie musicale». Dans De la sémiologie à la musique, Les cahiers du département d'études littéraires, $\mathrm{n}^{\circ} 10$, chap. 10. Montréal : Université du Québec à Montréal.

. 1990. "Arom, ou le sémiologue sans le savoir ». Analyse musicale, $\mathrm{n}^{0} 23: 77-82$.

1992a. « Existe-t-il des relations entre les diverses méthodes d'analyse? ». Dans Secondo Convegno di Analisi Musicale, sous la dir. de Rossana Dalmonte et Mario Baroni, 537-65. Trente : Università degli Studi di Trento.

_. 1992b. « Nicolas Ruwet musicologue ". Dans De la musique à la linguistique : hommages à Nicolas Ruwet, sous la dir. de Liliane Tasmowski et Anne Zribi-Hertz, 24-38. Ghent : Communication and Cognition.

1997a. «De la sémiologie générale à la sémiologie musicale : l'exemple de La cathédrale engloutie de Debussy ». Protée 25, $\mathrm{n}^{0} 2: 7-20$.

- 1997b. "Quelle est la pertinence de la théorie de Lerdahl-Jackendoff?». Dans Actes de la troisième Conférence internationale pour la perception et la cognition musicales, sous la dir. d'Irène Deliège, 254-57. Université de Liège, 23-27 juillet 1994. Trad. angl. dans Perception and Cognition of Music, sous la dir. d'Irène Deliège et John A. Sloboda, 413-19. Hove : Psychology Press, 1997.

. 1998. "Le solo de cor anglais de Tristan und Isolde, essai d'analyse sémiologique tripartite ». Musicae Scientiae, $\mathrm{n}^{0}$ spécial : 43-61.

2002. "Musica e significato ". Dans Enciclopedia della Musica, sous la dir. de Jean-Jacques Nattiez, vol. 2, Il sapere musicale, 206-38. Turin : Einaudi.

- 2003. "Structure, structuralisme et création musicale au XX $\mathrm{XX}^{\mathrm{e}}$ siècle ». Dans $L$ 'orizzonte filosofico del comporre nel ventesimo secolo / The Philosophical Horizon of Composition in the Twentieth Cemtury, sous la dir. de Gianmari Borio, 51-68. Venise : Il Mulino.

- 2004. «La signification comme paramètre musical ». Dans Musiques. Une Encyclopédie pour le XXI siècle, sous la dir. de Jean-Jacques Nattiez, 
vol. 2, Les savoirs musicaux, 256-89. Arles : Actes Sud / Cité de la musique. Vers. fr. de Nattiez 2002.

Nattiez, Jean-Jacques, et Louise Hirbour-Paquette. 1973. " Analyse musicale et sémiologie : à propos du Prélude de Pelléas ». Musique en Jeu, $\mathrm{n}^{0} 10$ : 42-69.

Naud, Gilles. 1975a. " Aperçus d'une analyse sémiologique de Nomos Alpha ». Musique en jeu, $\mathrm{n}^{0} 17: 63-72$.

- 1975b. «Le problème des transformations dans l'analyse musicale ». Semiotica 15, $\mathrm{n}^{0} 1: 28-32$.

Nettl, Bruno. 1958. "Some Linguistic Approaches to Musical Analysis ». Journal of the International Folk Music Council $10: 37-41$. Trad. fr. « De quelques méthodes linguistiques appliquées à l'analyse musicale ». $\mathrm{Mu}$ sique en jeu, $\mathrm{n}^{\circ} 5: 61-66$.

Noske, Fritz. 1977. « La segmentazione di un tema di Mozart ». Rivista italiana di musicologia 12, $\mathrm{n}^{0} 1: 130-35$.

Osmond-Smith, David. 1975. "Iconic Relations within Formal Transformations ». Dans Stefani 1975, 45-55. Trad. fr. " L'iconisme formel : pour une typologie des transformations musicales ». Semiotica $15, \mathrm{n}^{0} 1: 33-47$.

Pagnini, Marcello. 1974. Lingua e musica, proposta per un'indagine strutturalistico-semiotica. Milan : Il Mulino.

Parain, Brice. 1942. Recherches sur la nature et les fonctions du langage. Paris : Gallimard.

Pekkilä, Erkki. 1987. «Ideal Patterns in the Finnish juoksuvalssi : A Paradigmatic Segment Analysis ». Semiotica 66, nos 1-3 : 299-314.

Pelinski, Ramon. 1981a. «Pratique émique de substitutions intervalliques dans le chant personnel des Inuit du Caribou ». Dans La musique des Inuit du Caribou. Cinq perspectives méthodologiques, chap. 3. Montréal : Les Presses de 1'Université de Montréal.

- 1981b. "Esquisse d'une grammaire des chants personnels des Inuit du Caribou ". Dans La musique des Inuit du Caribou. Cinq perspectives méthodologiques, chap. 5. Montréal : Les Presses de l'Université de Montréal. Trad. angl. dans Baroni et Callegari 1984, 273-86.

Perlman, Alan, et Daniel Greenblatt. 1981. "Miles Davies Meets Noam Chomsky : Some Observations on Jazz Improvisation and Language Structure ». Dans Steiner 1981, 169-83.

Pike, Kenneth Lee. 1954. Language in Relation to a Unified Theory of the Structure of Human Behavior. Partie I. Éd. prélim., Glendale, Calif. : Summer Institute of Linguistics. Éd. compl., La Haye : Mouton, 1967.

Powers, Harold. 1980. « Language Models and Musical Analysis ». Ethnomusicology 24, $\mathrm{n}^{0} 1: 1-60$.

Ricœur, Paul. 1969. Le conflit des interprétations. Paris : Éditions du Seuil. Rosen, Charles. 1971. Classical Style. $2^{\mathrm{e}}$ éd. augm. New York : W. W. Norton, 1997. Trad. fr. de la $1^{\text {re }}$ éd., Le style classique. Paris : Gallimard, 1978.

Rothgeb, John. 1968. " Harmonizing the Unfigured Bass : A Computational Study ». Thèse de doctorat, Yale University. 
Roy, Stéphane. 2003. L'analyse des musiques électroacoustiques : modèles et propositions. Préface de Jean-Jacques Nattiez. Paris : L'Harmattan.

Ruwet, Nicolas. 1959. "Contradictions du langage sériel ». Revue belge de musicologie 13 : 283-97. Repris dans Ruwet 1972, 23-40.

- 1962. « Note sur les duplications dans l'œuvre de Claude Debussy ». Revue belge de musicologie $16: 57-70$. Repris dans Ruwet 1972, 70-99.

- 1963. "Linguistique et sciences de l'homme ». Esprit, n 322 : 564-78.

. 1966. "Méthodes d'analyse en musicologie ". Revue belge de musicologie 20 : 65-90. Repris dans Ruwet 1972, 100-34.

- 1967a. "Quelques remarques sur le rôle de la répétition dans la syntaxe musicale ». Dans To Honor Roman Jakobson, 1693-1 703. La Haye : Mouton. Repris dans Ruwet 1972, 135-48.

- 1967b. "Musicologie et linguistique ». Revue internationale des sciences sociales $19, \mathrm{n}^{0} 1: 85-93$.

-1972. Langage, musique, poésie. Paris : Éditions du Seuil.

- 1975. "Théorie et méthodes dans les études musicales ». Musique en jeu, $\mathrm{n}^{\circ} 17: 11-36$.

Sadai, Yizhak. 1986. " L'application du modèle syntagmatique-paradigmatique à l'analyse des fonctions harmoniques ». Analyse musicale, $\mathrm{n}^{0} 2$ : $35-43$.

Sadie, Stanley, dir. 1980. The New Grove Dictionary of Music and Musicians. 20 vol. Londres : Macmillan Publishers.

2001. The New Grove Dictionary of Music and Musicians, $2^{\mathrm{e}}$ éd. 29 vol. Londres : Macmillan Publishers.

Salzer, Felix. 1952. Structural Hearing : Tonal Coherence in Music. 2 vol. New York : Dover Publications.

Sampaio, Luiz Paulo de Oliveira. 1999. «Reflets dans le miroir. Essai d'analyse sémiologique tripartite des Variations, opus 27 de Webern ». Thèse de doctorat, Université de Montréal.

Sapir, J. David. 1969. " Diola-Fogny Funeral Songs and the Native Critic ». African Language Review 8 : 176-91.

Saussure, Ferdinand de. 1916. Cours de linguistique générale. Paris-Lausanne : Payot. Trad. it. et éd. crit. de Tullio de Mauro, Corso di linguistica generale. Bari : Laterza, 1967. Version fr. Paris : Payot, 1972.

Schaeffer, Pierre. 1966. Traité des objets musicaux. Paris : Éditions du Seuil.

Schneider, Reinhard. 1980. Semiotik der Musik : Darstellung und Kritik. Munich : Wilhelm Fink Verlag.

Schoenberg, Arnold. 1943. Models for Beginners in Composition. New York : Schirmer.

Schulte-Tenckhoff, Isabelle. 1999. "L'ethnomusicologie : structuralisme ou culturalisme? Entretien avec Jean-Jacques Nattiez ». Cahiers de musique traditionnelle 12 : 153-72.

Slawson. A. Wayne. 1985. Sound Colour. Los Angeles : University of California Press. 
Sloboda, John A. 1985. The Musical Mind. The Cognitive Psychology of Music. Oxford : Oxford University Press.

Smoliar, Stephen William. 1980. «A Computer Aid for Schenkerian Analysis ». Computer Music Journal 4, no 2 : 41-59.

Springer, George P. 1956. "Language and Music : Parallel and Divergencies ». Dans For Roman Jakobson, 604-13. La Haye : Mouton. Trad. fr. "Le langage et la musique : parallélismes et divergences ». Musique en jeu, $\mathrm{n}^{\circ} 5$ (1971) : 31-44.

Steedman, Mark J. 1984. "A Generative Grammar for Jazz Chord Sequences ». Music Perception 2, $\mathrm{n}^{0} 1: 53-78$.

Stefani, Gino, dir. 1975. Proceedings of the $1^{\text {st }}$ International Congress on Semiotics of Music (Beograd 1973). Pesaro : Centro di Iniziativa Culturale.

-1976. Introduzione alla Semiotica della Musica. Palerme : Sellerio.

Steiner, Wendy, dir. 1981. The Sign in Music and Literature. Austin : University of Texas Press.

Stravinsky, Igor. [1935-36] 1971. Chroniques de ma vie. Paris : MédiationsGonthier.

Sundberg, Johann, et Björn Lindblom. 1972. " A Generative Theory of Swedisch Nursey Tunes ». Dans Stefani 1975, 111-24.

- 1976. "Generative Theories in Language and Music Descriptions ». Cognition 4 : 99-122.

Sychra, Antonin. 1948. "Lidov písen shlediska semiologického ». Slova a Slovesnost $11: 7-23$. Trad. fr. " La chanson folklorique du point de vue sémiologique ". Musique en jeu, $\mathrm{n}^{0} 10: 12-33$.

Tagg, Philip, et Bob Clarida. 2003. Ten Little Title Tunes. New York et Montréal : The Mass Media Music Scholars' Press.

Troubetzkoy, Nikolai S. 1939. Grundzüge der Phonologie. Prague : Travaux du Cercle linguistique de Prague, vol. 7. Trad. fr. Principes de phonologie. Paris : Klincksieck, 1949.

Vaccaro, Jean-Michel. 1975. « Propositions d'analyse pour une polyphonie vocale du XVI ${ }^{\mathrm{e}}$ siècle ». Revue de musicologie 61, $\mathrm{n}^{0} 1: 35-58$.

Varèse, Edgar. [1925] 1983. Écrits. Paris : Christian Bourgois éditeur.

Wenk, Arthur B. 1988. «Parsing Debussy : Proposal for a Grammar of his Melodic Practice ». Musikometrika $1: 237-56$, "Quantitative Linguistics 》.

Winograd, Terry. 1968. "Linguistics and the Computer Analysis of Tonal Harmony ». Journal of Music Theory 12, $\mathrm{n}^{0} 1: 2-49$.

\section{Résumé}

Au cours de la seconde moitié du $\mathrm{XX}^{\mathrm{e}}$ siècle, l'analyse musicale s'est tournée vers les modèles linguistiques d'inspiration structuraliste pour tenter de renouveler et de rendre plus explicites les méthodes traditionnelles. Dans cet article, qui est à la fois une bibliographie critique et un bilan épistémologique, JeanJacques Nattiez examine dans quel contexte musicologie et linguistique se sont 
rencontrées. Il présente tour à tour les applications du modèle phonologique, du modèle paradigmatique (avec une discussion des propositions et de la postérité de Ruwet) et les diverses grammaires musicales descriptives d'inspiration génératives qui ont été proposées. Il examine aussi bien les travaux qui portent sur la musique occidentale que sur les répertoires qu'étudient l'ethnomusicologie et les spécialistes de musique populaire.

\begin{abstract}
Throughout the second half of the twentieth century, musical analysis has turned for structuralist inspiration to linguistic models in order to rejuvenate and make traditional methods more explicit. In this article, which is both a critical bibliography and an epistemological claim, Jean-Jacques Nattiez examines the contexts in which musicology and linguistics intersect. He presents various applications of phonological models, paradigmatic models (with a discussion of the work and legacy of Ruwet), and various descriptive musical grammars of generative thought that have been proposed. He examines works not only from the Western canon but also ethnomusicological and popular music repertoires.
\end{abstract}

